Utah State University

DigitalCommons@USU

$12-2017$

\title{
Optimizing Bioengineered Vascular Systems: A Genetic Algorithm Approach
}

Sima Mehri Kochaki

Utah State University

Follow this and additional works at: https://digitalcommons.usu.edu/etd

Part of the Computer Sciences Commons

\section{Recommended Citation}

Kochaki, Sima Mehri, "Optimizing Bioengineered Vascular Systems: A Genetic Algorithm Approach" (2017). All Graduate Theses and Dissertations. 6693.

https://digitalcommons.usu.edu/etd/6693

This Thesis is brought to you for free and open access by the Graduate Studies at DigitalCommons@USU. It has been accepted for inclusion in All Graduate Theses and Dissertations by an authorized administrator of DigitalCommons@USU. For more information, please contact digitalcommons@usu.edu.

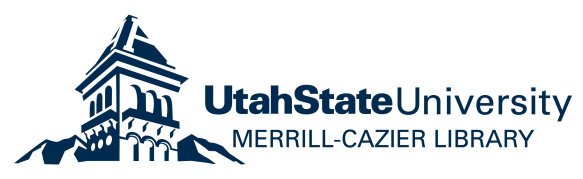




\title{
OPTIMIZING BIOENGINEERED VASCULAR SYSTEMS: \\ A GENETIC ALGORITHM APPROACH \\ by
}

Sima Mehri Kolchaki

A thesis submitted in partial fulfillment

of the requirements for the degree

\author{
of \\ MASTER OF SCIENCE
}

in

Computer Science

Approved:

Nicholas S. Flann, Ph.D.

Vicki Allan, Ph.D.

Major Professor

Committee Member

Vladimir Kulyukin, Ph.D.

Mark R. McLellan, Ph.D.

Vice President for Research and

Committee Member

Dean of the School of Graduate Studies

\section{UTAH STATE UNIVERSITY \\ Logan, Utah}


Copyright (c) Sima Mehri Kolchaki 2017

All Rights Reserved 


\author{
ABSTRACT \\ Optimizing Bioengineered Vascular Systems: \\ A Genetic Algorithm Approach \\ by \\ Sima Mehri Kolchaki, Master of Science \\ Utah State University, 2017
}

Major Professor: Nicholas S. Flann, Ph.D.

Department: Computer Science

Efficient metabolism in bioengineered tissues requires a robust vascular system to provide healthy microenvironments to the cells and stroma. Such networks form spontaneously during embryogenesis from randomly distributed endothelial cells. There is a need to bioengineer endothelial cells so that network formation and operation is optimal for synthetic tissues. This work introduces a computational model that simulates de novo vascular development and assesses the effectiveness of the network in delivering nutrients and extracting waste from tissue. A genetic algorithm was employed to identify parameter values of the vaculogenesis model that lead to the most efficient and robust vascular structures. These parameter values control the behavior of cell-level mechanisms such as chemotaxis and adhesion. These studies demonstrate that genetic algorithms are effective at identifying model parameters that lead to near-optimal networks. This work suggests that computational modeling and optimization approaches may improve the effectiveness of engineered tissues by suggesting target cellular mechanisms for modification.

(45 pages) 
PUBLIC ABSTRACT

\author{
Optimizing Bioengineered Vascular Systems: \\ A Genetic Algorithm Approach \\ Sima Mehri Kolchaki
}

Efficiency of current cell cultures producing biological products is limited due to accumulation of the product and waste on the cells. A previous work aims at assisting bioengineers in solving this problem by introducing a new set of cells which possess the ability to connect to one another and thus form a network. Once created, the network of cells can help the initial cellules by removing their waste and product as well as nourishing them. Our project explores a technique to be able to create the best network of such cells; hence maximizing the amount of metabolic product in the cell culture. 


\section{DEDICATION}

To my lovely parents and my husband, Mohammad

without whom, I would never be who I am 


\section{ACKNOWLEDGMENTS}

I would first like to thank my thesis advisor Dr. Nicholas Flann who was always so kind and supportive, like a father. Without his continuous assistance and cooperation, I would never be able to do my graduate work this smoothly.

I would also like to thank my other committee members, Dr. Vicki Allan and Dr. Vladimir Kulyukin. I am gratefully indebted to their valuable presence when I needed them and their comments on this thesis.

Finally, I must express my very profound gratitude to my parents and especially to my loving husband, Mohammad, for providing me with unfailing support and continuous encouragement throughout my years of study and through the process of researching and writing this thesis. This accomplishment would not have been possible without them. Thank you.

Sima Mehri Kolchaki 


\section{CONTENTS}

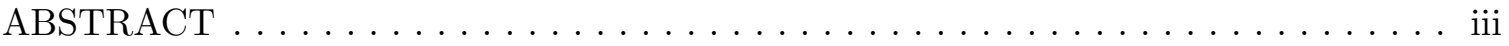

PUBLIC ABSTRACT $\ldots \ldots \ldots \ldots \ldots \ldots \ldots \ldots \ldots \ldots \ldots \ldots \ldots$

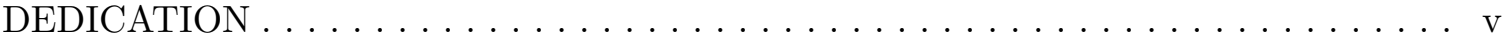

ACKNOWLEDGMENTS . . . . . . . . . . . . . . . . . . . vi

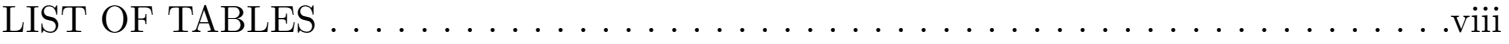

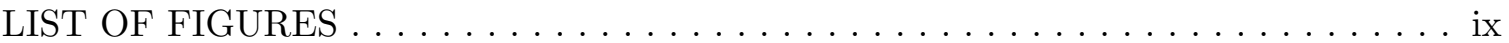

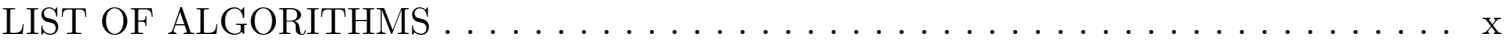

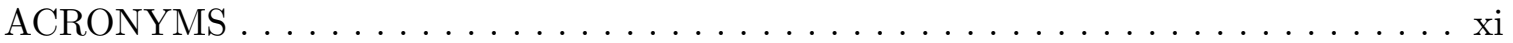

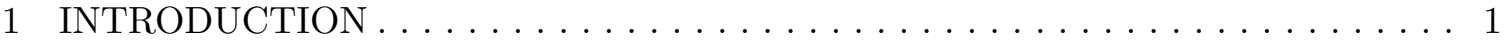

1.1 Biological Background . . . . . . . . . . . . . . . . . . . 1

1.2 Optimization . . . . . . . . . . . . . . . . . 2

1.3 Genetic algorithms . . . . . . . . . . . . . . . . . . 4

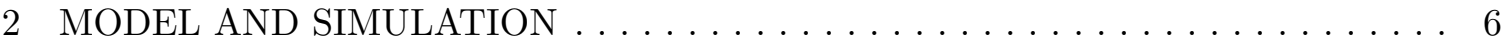

2.1 Self Organizing Vascularization Model . . . . . . . . . . . . . . . . . . . 6

2.2 De Novo Vascularization Model . . . . . . . . . . . . . . . . . . . . . 7

2.3 Network Structure Evaluation . . . . . . . . . . . . . . . . . . 10

2.4 Network Fitness Evaluation . . . . . . . . . . . . . . . . . . . . 11

3 IMPLEMENTATION AND EXPERIMENTAL SET-UP . . . . . . . . . . . . . . 14

3.1 Genetic Algorithm Implementation . . . . . . . . . . . . . . . . . . . 14

3.2 Experimental Set-up . . . . . . . . . . . . . . . . . . . 18

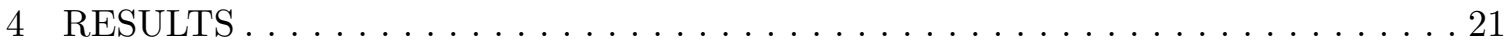

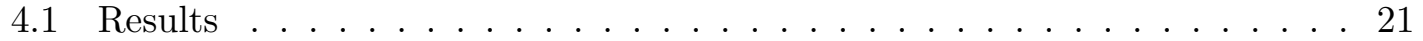

$4.1 .1 \quad$ First Experiment . . . . . . . . . . . . . . . . . . 21

4.1 .2 Expanded Experiment . . . . . . . . . . . . . . . . 24

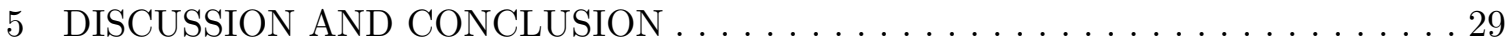

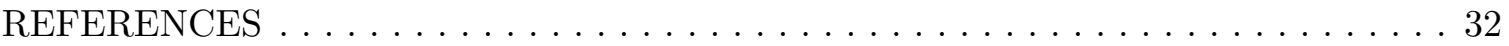




\section{LIST OF TABLES}

Table

Page

2.1 Known parameter descriptions . . . . . . . . . . . . . . . 8

3.1 Parameters searched by the GA . . . . . . . . . . . . . 15

3.2 Expanded parameters searched by the GA . . . . . . . . . . 15

4.1 The best parameter values found by the GA in initial experiment . . . . . . 21

4.2 Parameter table corresponding to cell cultures depicted in Figures 4.2 and 4.322

4.3 The best parameter values found by the GA . . . . . . . . . . 25

4.4 Parameter table corresponding to cell cultures depicted in Figures 4.6 . . . . 27 


\section{LIST OF FIGURES}

Figure

1.1 Architecture of a genetic algorithm depicting the genetic operator steps until

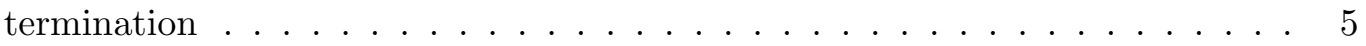

2.1 Self-organizing network . . . . . . . . . . . . . . . . 9

2.2 Cell culture activation . . . . . . . . . . . . . . . . . . 13

3.1 A sample graph during pruning . . . . . . . . . . . . . . . 17

3.2 A closer look at graph pruning . . . . . . . . . . . . . . . 18

4.1 Cell culture production improvement over generations of the GA in the first

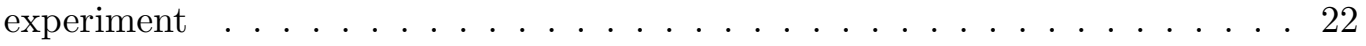

4.2 Sample results of the GA during its evolution . . . . . . . . . . 23

4.3 A sample of best solutions identified by the genetic algorithm during evolution 24

4.4 A visualization of cell culture productivity improvement over generations of the genetic algorithm in the expanded experiment . . . . . . . . 25

4.5 Two of the best solutions identified by the genetic algorithm during evolution. 26

4.6 Some solutions unsuccessful at forming a reliable network of cells . . . . . . 28 


\section{LIST OF ALGORITHMS}

Algorithm $\quad$ Page

3.1 Steps at each GA iteration . . . . . . . . . . . . . . . . 14

3.2 Graph pruning steps . . . . . . . . . . . . . . . . . 16 


\section{ACRONYMS}

$\begin{array}{ll}\text { GA } & \text { Genetic Algorithm } \\ \text { jGAP } & \text { Java Genetic Algorithms Package } \\ \text { EPS } & \text { Extracellular Polymeric Substances }\end{array}$




\section{CHAPTER 1}

INTRODUCTION

\subsection{Biological Background}

Multicellular organisms depend on vascular systems for nutrient delivery and waste removal [1]. These vascular networks are formed either through vasculogenesis, a biological process in which scattered vessel precursor cells self-organize to create new networks or through angiogenesis, in which new vessels sprout from the existing vessels.

Both vasculogenesis and angiogenesis are driven primarily by chemotaxis, a mechanism in which cells move in response to a chemical gradient, along with cell-cell adhesion [2]. While many questions remain, progress in understanding and exploiting both vasculogenesis and angiogenesis is being made from a bioengineering perspective [3] [4]. Dahl et al. [5] successfully implanted tissue-engineered vascular grafts in baboons and dogs. Melero-Martin et al. [6] showed that robust development of functional vascular networks is possible in vivo.

With additional research in this area, bioengineered cells could be used to form functional vascular networks to create a useful delivery mechanism in synthetic tissues. However, to apply bioengineering approaches to vascular cells, genetic targets need to be identified that when modified, improve the effectiveness of the vascular systems that emerge. Given the vast number of possible targets, a method is required that could assist engineers in identifying those genetic targets.

Genetic algorithm (GA) is widely used in optimization problems. It is a metaheuristic derived from natural selection in biology; an evolutionary algorithm which finds high-quality solutions using methods such as mutation, crossover and selection. Such operators originate from biological processes.

This paper presents a proof-of-concept genetic algorithm approach to solving this problem. The genetic algorithm acts to modify a computational model of vascular network 
development embedded within a cell cultivation environment. The search space of the optimization are the values of model parameters which control the mechanisms of the vascular cells such as chemotaxis. Changing these parameters modifies the behavior of individual cells which ultimately influence the spatial organization of the emergent vascular network. To evaluate the quality of this network, the model simulates its operation by determining fluid flow through each vessel, and subsequent nutrient delivery to the cultured cells. The returned fitness value quantifies the total metabolic activity of culture by using bioengineered microbial cells and measuring the total product produced.

The rest of the paper is organized as follows. First, an introduction to optimization problem solutions and Genetic Algorithms is presented. Next, the computational model of de novo vascularization is described. The third chapter describes the details of the genetic algorithm implementation. Later in the paper, we present the results which show that genetic algorithms can identify parameter values that improve network quality. The paper concludes with a discussion of the potential impact of this work and the remaining challenges which need to be solved to operationalize the technique.

\subsection{Optimization}

Finding the best possible solution to a given problem among all feasible solutions is called optimization. Optimization, as a powerful modeling and problem solving methodology, has a broad range of applications in management science, industry and engineering [7]. This tool facilitates decision making and analyzing physical systems.

The first step towards using optimization is constructing a model in which the mathematical concepts of variables, objective(s) and constraints are determined. Variables, also referred to as unknowns, represent the components of the system for which values are needed. An instance to them can be the amount of resources used in some physical applications. The second parameter of the model is the objective; meaning the optimal result the system should be searching for. The objective is the main goal in optimization problems which needs to be either maximized or minimized. Constraints contribute to the last required component. They define any requirements on the variables including value ranges 
or other specifications.

Depending on the type of problem, different optimization techniques are required. Problem types vary based on variable, problem and data characteristics as well as number of objectives.

The models in which variables are continuous, meaning that they can take any real values, are called continuous optimization models. On the other hand, in case only specific values such as discreet integers or a subset of them, can be assigned to the variables, a discreet optimization model should be deployed.

Another determining factor in choosing a model is existence of constraints. Any sort of limitation on the model variables including value range and equality or inequality systems of relationships can change the optimization model from an unconstrained to a constrained one. Although most problems possess one or even multiple objectives, there are cases where no such a function is required. For instance, feasibility problems are problems in which the goal is to find values for the variables that satisfy the constraints of a model with no particular objective to optimize.

Our study targets to optimize a vascular system contained in a cellular culture by maximizing the amount of possible metabolic product. The bio-model specifications are inserted into an XML file with various parameters controlling the behavior of the system. In this case, certain parameter values found inside the mentioned XML file are the variables of the mathematical model. The objective function is the maximization of cellular product and there are some constraints on the variable values. These constraints include unique ranges for the variables which are set heuristically in order to decrease GA operation time.

Since the target parameters in our project can take any real number values in a certain range, the model will be a continuous constrained one. A single maximization function is the objective of the optimization model while it does not suffer any uncertainties.

While there are many algorithms available for solving optimization problems, not many can be used for a specific one due to the required model type. Genetic and evolutionary algorithms are recognized as a technique to find high-quality solutions to optimization 
problems. They offer one way to find "good" solutions to problems pertaining to nonsmooth optimization known as the most difficult problem type.

\subsection{Genetic algorithms}

A genetic algorithm (GA) applies the principles of evolution found in nature to the problem of finding an optimal solution to a problem. In a genetic algorithm, the problem is encoded in a series of bit strings that are manipulated by the algorithm and the decision variables and problem functions are used directly.

GAs keep a population while evolving it over time. The population consists of various chromosomes which in turn, comprise genes. The parameter values which are being searched for are encoded in genes. Therefore, a chromosome consists of one or several parameters which need correct values.

The fitness of a chromosome is assessed by the desired parameter which is defined by the user. At each iteration, it is calculated for every chromosome present in the population. When the GA receives fitness values of all its population, based on some operators, it creates the new population. The operators are selection, mutation and cross-over. The new population can include the ones which were already present and/or new ones.

Inspired by the role of mutation of an organism's DNA in natural evolution, a GA periodically makes random changes or mutations in one or more members of the current population, yielding a new candidate solution. This does not guarantee a better chromosome; however, it increases the randomness of the algorithm. Another genetic operator is cross over which is a process of taking more than one parent solutions and producing a child solution from them. The last operator, selection, is applied after the other two. Using this, the GA decides which members of the population to keep for later breeding. Figure 1.1 depicts the process a GA follows until termination.

A drawback of any evolutionary algorithm is that it has no test to determine whether a solution is optimal. This is due to the fact that the solutions are compared to other ones that the algorithm has already tried. This also means that the stopping point of 


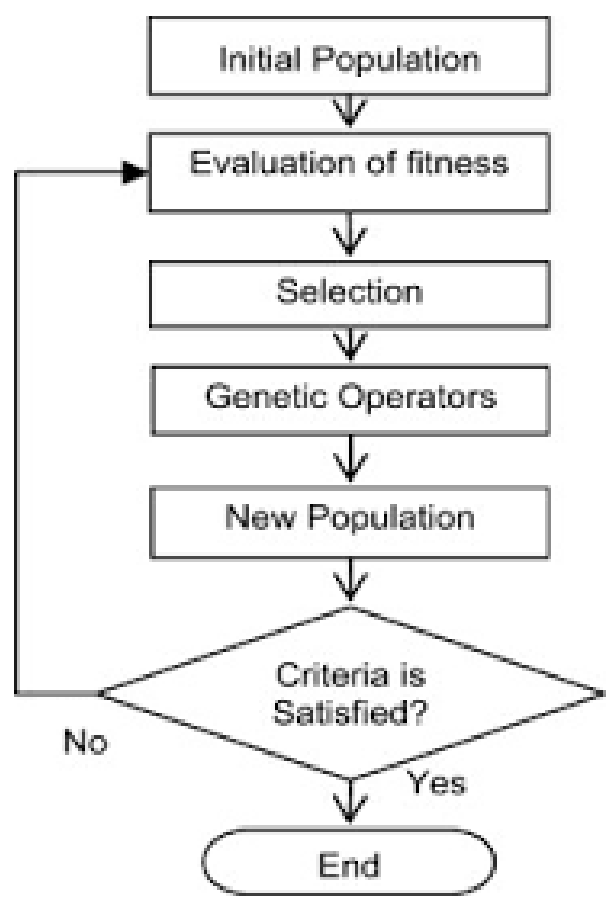

Fig. 1.1: Architecture of a genetic algorithm depicting the genetic operator steps until termination

an evolutionary algorithm can only be the length of time, or the number of iterations or candidate solutions, that it is set to explore in the beginning. 


\section{CHAPTER 2}

\section{MODEL AND SIMULATION}

A bio-reactor modeling package called cDynomics was used in this study (See section 3.1 for more details). Following, details of the adopted model will be presented.

A confined space called "world" represents the cell culture which can take the form of a rectangle, circle, etc. Its bordering surfaces can act differently when facing various solutes and particles regarding permeability. The resolution also can be adjustable with the minimum amount of $1 \mu \mathrm{m}$.

The world, contains spherical 2D or 3D objects representing bacterial cells. Cells also have specific traits such as bio mass, division, death, shoving, etc. While they are allowed to freely move inside the world, certain rules control their behavior, like intercellular attraction, repulsion and tight junction formation. In addition, cells can secrete different types of chemoattractants to interact with others.

Other components within the world are solutes which model various solutions inside cell cultures like nutrients. In the end, a set of interactions between components of the system is defined that determines the event when two components reach each other during the simulation.

\subsection{Self Organizing Vascularization Model}

At this proof-of-concept stage, a two-dimensional model was constructed and evaluated. Figure 2.1(a) illustrates the initial state of the simulated cell culture. Vessel cells (blue) that simulate the external circulatory system are arranged in two columns on both sides of the cell culture area. The left column represents the source, and the right column represents the sink, in parallel to the arterial-venous network architecture in vertebrates [8].

The tissue to be supported by the vascular network is simplified to be a field of identical cells, referred to as supported cells (yellow), randomly distributed across the area in between 
the two circulatory cell columns. Mixed within this field of supported cells are vascular cells (red), similar to the endothelial cells in vertebrates [2]. It is the mechanisms of the vascular cells that are to be optimized by the genetic algorithm.

During simulation, the randomly distributed vascular cells self-organize and can form a vascular network connected to both columns of circulatory cells. If this process succeeds, the supported cells will form clusters contained within each network lacuna. An example of a self-organized network is illustrated in Figure 2.1(b). This developmental process is described in more detail in Section 2.2.

Then, the performance of the network is evaluated (refer to section 2.3 ), followed by an operation simulation. As the network provides nutrients, the supported cells become active (turn green in the figures) and consume nutrients and secrete wastes. Note that in this case the network fitness would be high because almost all of the supported cells are active and close to a vessel. This network operational process is described in more detail in Section 2.4.

\subsection{De Novo Vascularization Model}

The two key cellular mechanisms in vascularization are chemotaxis, where cells move in respond to chemical gradients, and tight junctions, where adjacent cells form strong bonds. Cells are modeled as particles that can secrete and respond to chemicals and move in response to forces. The modeling framework is based on iDynamics [9] originally created for bio-film models.

Initially, the particles representing epithelial cells are placed randomly in the simulation domain mixed with the support cells. A chemotactic nutrient is initially supplied at concentration $N_{c}$, set at $8.9 \times 10^{-8} \mathrm{~m}^{-2}$. The fixed cells on the sides of the cell culture secrete $C_{\text {long }}$, a chemoattractant with small decay rate, and $C_{\text {short }}$, a chemoattractant with a large decay rate. The moving cells within the culture only secrete $C_{\text {short }}$. The decay rates affect the shape of the chemoattractant gradient. $C_{\text {short }}$ is a localized concentration with a sharp gradient, and $C_{\text {long }}$ has a long-distance shallow gradient. The concentrations of each are described by the Monod-kinetic reaction in Equation 2.1. $D_{c}$ of both chemoattractants 
are set to $1 \times 10^{-13} \mathrm{~m}^{2} \mathrm{~s}^{-1}$ as given in the in-vitro angiogenesis study of Merks et al [2]; $\beta$ is the decay rate; $M$ is the mass of the secreting cell; $k$ is the Monod-kinetic coefficient; $\mu$ is the maximum secretion rate.

$$
\frac{\partial C}{\partial t}=D_{c} \nabla^{2} C+\mu \frac{N_{c}}{\left(N_{c}+k\right)} M-\beta C
$$

The moving cells respond to the gradient of the chemoattractants by tending towards higher concentrations in a process described in Equation 2.1 and by Adler [10]. Let $p$ be a particle that responds to chemoattractant $C$. A random unit vector $\vec{c}$ is generated and considered as a potential chemotactic force on $p$. The local gradient of chemoattractant across $p$ in direction $\vec{c}$ is determined by sampling $C$ ahead of $p$, referred to as $C^{+}$, and behind $p$, referred to as $C^{-}$. The magnitude of force $F$ in direction $\vec{c}$ is given by the equation $2.2[2]$, where $\lambda$ is the parameter that controls the magnitude of the response to the gradient and $\alpha$ controls saturation of the chemotactic force. The force $F$ is only applied to the particle if greater than zero.

$$
F=\lambda\left(\frac{C^{+}}{1+\alpha C^{+}}-\frac{C^{-}}{1+\alpha C^{-}}\right)
$$

Adhesive forces act among the particles and are attractive at close distances and neutral otherwise. In this work, endothelial cell-cell adhesion is strong and endothelial-supported cell is weak. When endothelial cells become close, tight junctions begin to form, binding the cells together as a precursor to vessel construction. In the model, if two adjacent cells form a tight junction, then a stiff spring connects the corresponding particles.

Table 2.1: Known parameter descriptions

\begin{tabular}{lll}
\hline Parameter & Value & Description \\
\hline \hline$D_{c}$ & $1 \times 10^{-13} \mathrm{~m}^{2} \mathrm{~s}^{-1}$ & Diffusion coefficient \\
$N_{c}$ & $3 \times 10 \mathrm{gL}^{-1}$ & Chemoattractant nutrient concentration \\
$M_{\text {fixed }}$ & $1 \times 10^{-11} \mathrm{~g}$ & Mass of fixed epithelial cells \\
$M_{\text {moving }}$ & $1 \times 10^{-11} \mathrm{~g}$ & Mass of moving epithelial cells \\
$\alpha$ & 0.1 & Saturation of chemotactic force \\
\hline
\end{tabular}




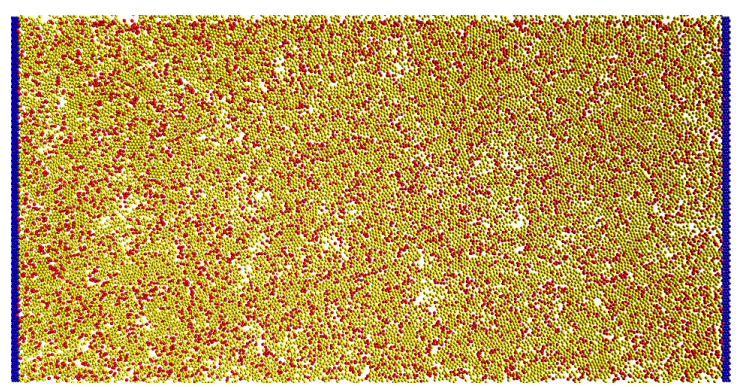

(a) Initial random distribution

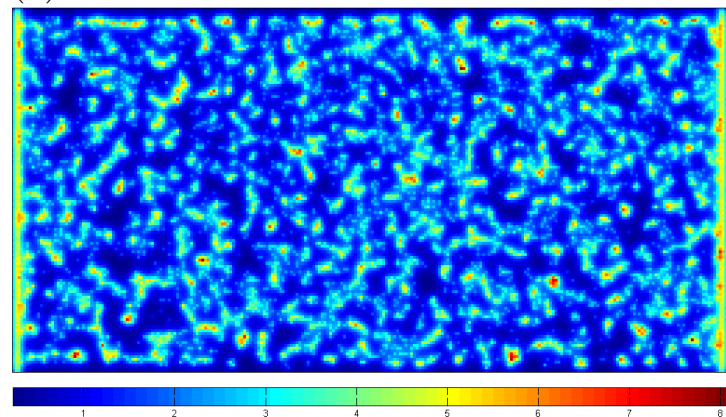

(c) Chemoattractant during formation

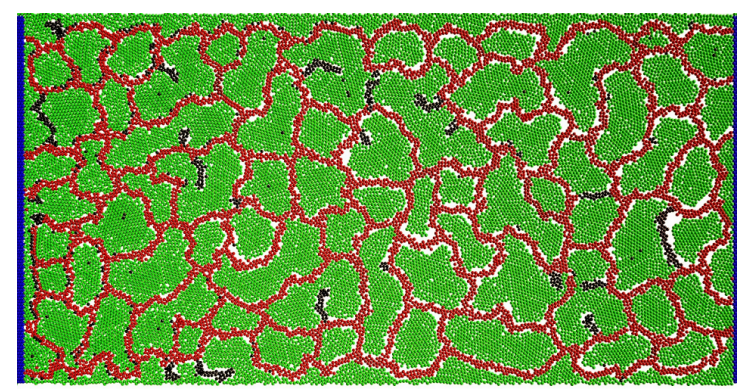

(b) Stable vascular network

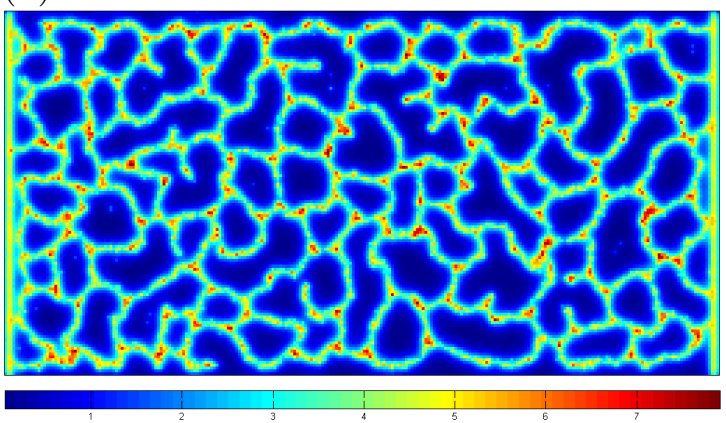

(d) Chemoattractant state distribution

Fig. 2.1: Self-organizing Network: (a) is the initial conditions with fixed circulatory cells (blue) on each side and vascular (red) and supported cells (yellow) randomly mixed; (b) is the steady state network following self organization; (c) is the distribution of chemoattractant during vessel formation and $(\mathrm{d})$ is at a steady state. In all images of biochemical distribution, blue signifies a low concentration, while red signifies a high concentration.

In addition to chemotactic force $F \cdot \vec{c}$, each particle experiences forces due to nonoverlapping constraints caused by competition for space. Once the net forces have been assigned to each particle, the system is relaxed by a shoving algorithm which moves the particles along their force vectors to minimize stress. In this way, the vessel particles push through the supported particles, form clumps due to attractive chemotactic forces, and then buckle and extend immature vessels. The system can eventually reach the morphology illustrated in Figure 2.1(b), in which all biomechanical forces are relaxed, and concentrations of molecules are stable, see Figure 2.1(d). 


\subsection{Network Structure Evaluation}

Once the vascularization phase is over, the vascular network is evaluated for determination of its ability in moving bio-products in and out of the simulated cell culture. An important feature is the connectivity of the vascular cells to both left and right pipe cells which insures flow through the network.

In order to estimate the flow, a graph was fit to the vascular network, where the location of cells were marked with nodes and the neighboring nodes were connected via edges. Section 3.1 covers the details of simplifying the graph for enabling flow calculation. For every node, edge and lacunae in the graph, a series of equations are generated using Poiseuilles Law, which are then solved for obtaining vessel flows.

In the end, the amount material flow is determined for every edge existing in the graph. First, a graph traversal is performed to determine if there exists a path through the network connecting the source and sink in Figure 2.1. Each node i in the graph is assigned an unknown variable $P_{i}$ representing the pressure at node i. Each edge (i, j) in the graph is assigned an unknown variable $Q(i, j)$ representing the flow through $\mathrm{i}, \mathrm{j}$, and an unknown variable $\Delta P_{(i, j)}$ representing the pressure drop $P_{i}-P_{j}$. Next a series of equations of the graph are generated using Poiseuilles Law [11] as given in Equations 3, 4 and 5 relating $Q_{(i, j)}, \Delta P_{(i, j)}$ and $P_{i}$ to characteristics of the network (r and the radius and length of each vessel) and operating conditions ( $P_{1}$ and $P_{n}$, the source and sink pressure respectively). For this simulation $\Delta P_{(1, n)}$ was set at 1 . The viscosity of the fluid $\eta$ is set to that of water at $25^{\circ} \mathrm{C}$. The following linear equations are generated: For each edge i, j:

For each edge i, $\mathrm{j}$ :

$$
Q_{(i, j)}=\frac{\pi r^{4} \Delta P_{(i, j)}}{8 \eta l}
$$

For each node, i with $\sigma(i)$ neighbors:

$$
\sum_{k \in \sigma(i)}^{\infty} Q_{(i, k)}=0
$$


For each lacunae cycle $\phi(k)$ :

$$
\sum_{(i, j) \in \phi(k)}^{\infty} \Delta P_{(i, j)}=0
$$

Finally, these equations are solved using a linear equation solver to calculate the flow rate $Q_{(i, j)}$ through all the vessels and pressure drop $\Delta P_{(i, j)}$ over each vessel [12].

\subsection{Network Fitness Evaluation}

The quality of the network is evaluated by determining the flow through each branch and modeling the release of nutrients and the capture of secretions from the supported cells. To assess the health of the supported cells given a running vascular network, the rate of metabolism of each supported cell is estimated by measuring how efficiently the cells produce some arbitrary product, $X$, described by Equation 2.6.

$$
\frac{\partial X}{\partial t}=+\mu_{p} \frac{N}{\left(N+k_{p}\right)} \frac{k_{i}}{\left(X+k_{i}\right)} M_{p}+D_{p} \nabla^{2} X
$$

Where $M_{p}$ is supported cell biomass. To produce a realistic model of cellular metabolism, the parameter values of the supported cells is replicated from [13] and is based on vanillin production of Pycnoporus cinnabarinus. The product $X$ is secreted by the supported cells consuming nutrient $N$ following Michaelis-Menten kinetics with a reaction rate of $\mu_{p}$, where the saturation of enzymes involved in the $X$ production is considered $k_{p}$. The negative feedback due to product inhibition is also taken into account, with a correspondent inhibitor constant $k_{i}[14],[15]$ and [16].

Nutrient will be consumed by the supported cells in direct correspondence to the production of $X$, but at a different reaction rate $\mu_{n}$ :

$$
\frac{\partial N}{\partial t}=-\mu_{n} \frac{N}{\left(N+k_{p}\right)} \frac{k_{i}}{\left(k_{i}+X\right)} M_{p}+D_{p} \nabla^{2} N
$$

Figure 2.2 illustrates the distribution of nutrient and product during simulated network operation for the network illustrated in Figure 2.1(c). Figure 2.2a illustrates the nutrient 
distribution before the supported cells have become active. In Figure $2.2 \mathrm{~b}$ gives the nutrient distribution following supported cell activation. In Figure 2.2c the distribution of the product is illustrated. Note the regions of low product (the blue areas) where the vascular flow is limited.

The final step is to integrate the amount of $X$ extracted from the cellular culture by the vascular network over a fixed period, in this case two hours. This accumulation of product will be a function of the aggregate metabolic rate of the supported cells. It is this value the simulator returns as the fitness score of the vascular network architecture. 


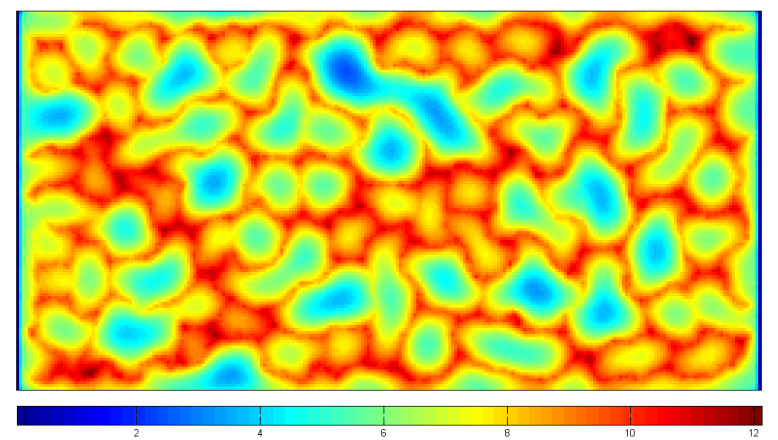

(a)

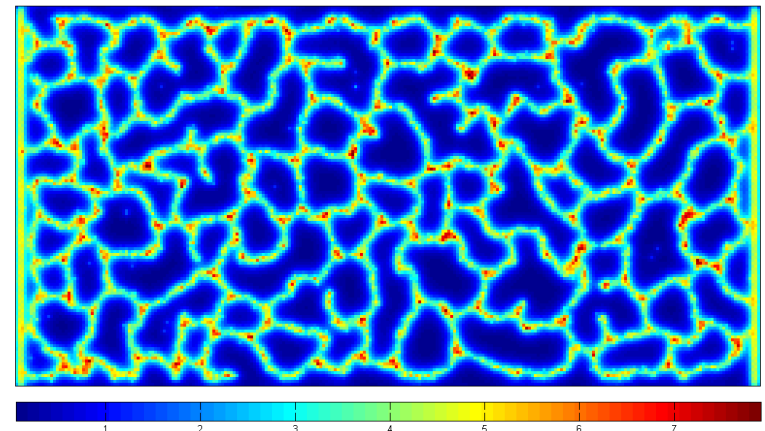

(b)

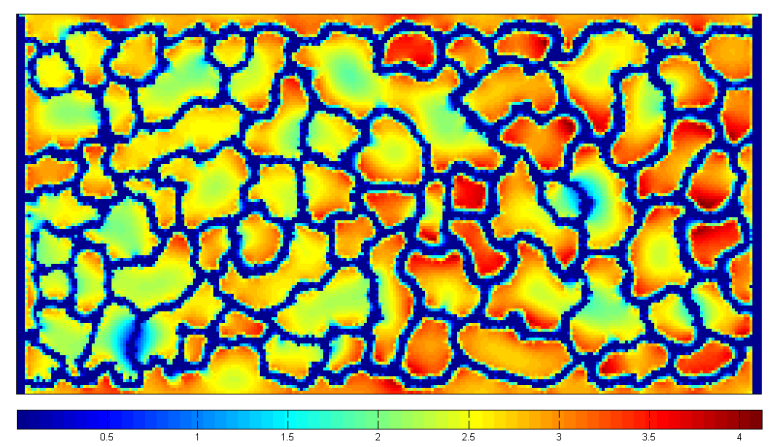

(c)

Fig. 2.2: Cell culture activation: The distribution of nutrient (a) when the vessel flow begins, but the supported cells are not yet active, (b) at steady state nutrient distribution when the supported cells are active; note that all extra-vessel nutrient is consumed, (c) the product distribution when the supported cells are active. 
CHAPTER 3

IMPLEMENTATION AND EXPERIMENTAL SET-UP

\subsection{Genetic Algorithm Implementation}

Java Genetic Algorithm Package (jGAP) [17] was used in this study for the GA implementation. It provided basic genetic mechanisms which were integrated to our solution.

After initial settings such as declaration of genes, chromosomes, population size and number of iterations, the GA would start the evolution process. Since the amount of metabolic product would determine the fitness of the current chromosome, GA evolution involved simulating the cellular culture followed by an evaluation of the vascular network and product calculation at each iteration. Algorithm 3.1 shows the steps during each GA iteration. Because GAs lack the ability to recognize the optimal solution, a number of iterations was set to mark termination point.

The input to this part was an XML file called the "protocol" which contained various parameters. The parameters included simulation modes, specifications of the world such as the size, details on solutions and the particles inside the model. Among all parameters inside this file, seven parameters which we believed were crucial to the formation of efficient

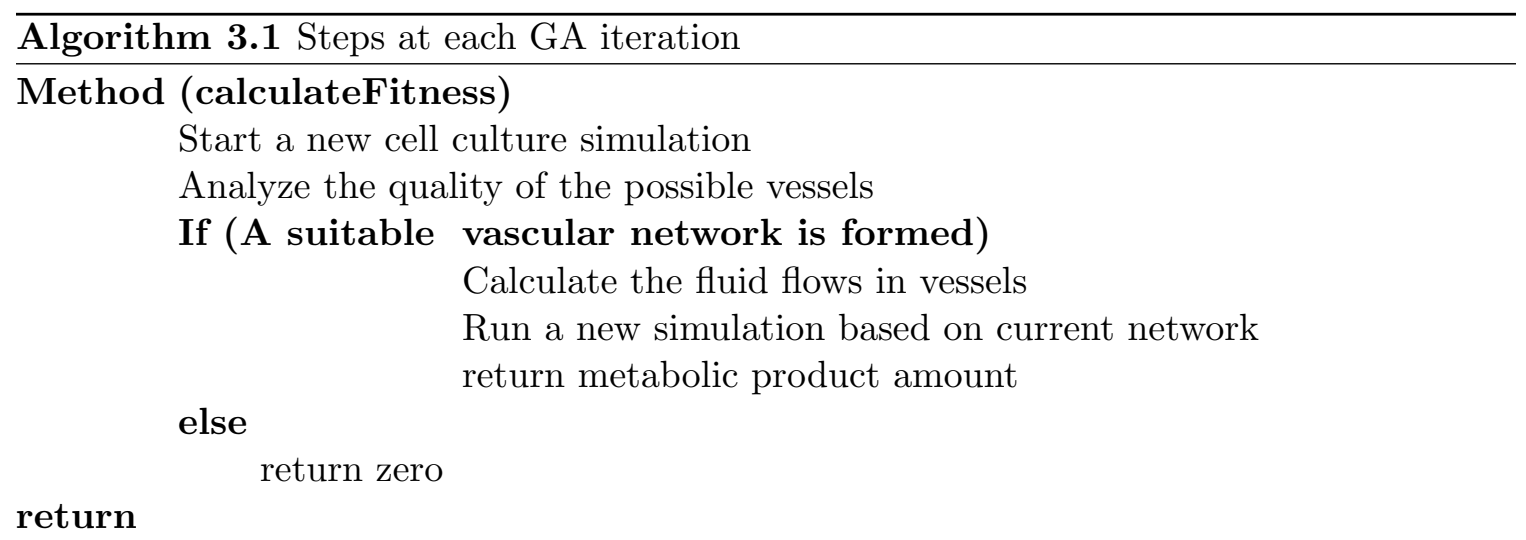


vascular networks (Table 3.1), were selected as GA variables. Later, we performed a second experiment with parameters expanded from seven to ten (Table 3.2).

The variables for changing simulation output were also set in the protocol XML file. Based on such settings, output files were created for user information. They included XML files containing information on each cell in the world. Examples of such information include spatial data, biomass, birth ID, generation, etc. Moreover, amount of different existing solutes and summary of cell status was output. Other interesting output included POVRay files which provided visual information about the simulation status.

After the initial simulation was done, the last XML file of cell status and the protocol file were input to the second phase which first evaluated the performance of the vascular network. In order to estimate the flow, a graph was fit to the vascular network, where the location of cells were marked with nodes and the nodes in a small proximity were connected via edges. The proximity threshold for connection through edges was set heuristically and then examined and improved by trial and error. The original graph was too complicated

Table 3.1: Parameters searched by the GA

\begin{tabular}{llll}
\hline Parameter & Low & High & Description \\
\hline \hline$k$ & $10^{-10}$ & $10^{-2}$ & Monod-kinetic coefficient \\
$\mu_{\text {short }}$ & 1 & 7 & Secretion rate of $C_{\text {short }}$ \\
$\mu_{\text {long }}$ & 1 & 7 & Secretion rate of $C_{\text {long }}$ \\
$\beta_{\text {short }}$ & $10^{-5}$ & $10^{-1}$ & Decay rate of $C_{\text {short }}$ \\
$\beta_{\text {long }}$ & $10^{-10}$ & $10^{-3}$ & Decay rate of $C_{\text {long }}$ \\
$\lambda_{\text {short }}$ & 1 & 6 & Chemoattractant response to $C_{\text {short }}$ \\
$\lambda_{\text {long }}$ & 1 & 6 & Chemoattractant response to $C_{\text {long }}$ \\
\hline
\end{tabular}

Table 3.2: Expanded parameters searched by the GA

\begin{tabular}{llll}
\hline Parameter & Low & High & Description \\
\hline \hline$k$ & $10^{-10}$ & $10^{-2}$ & Monod-kinetic coefficient \\
$\mu_{\text {short }}$ & 1 & 7 & Secretion rate of $C_{\text {short }}$ \\
$\mu_{\text {long }}$ & 1 & 7 & Secretion rate of $C_{\text {long }}$ \\
$\beta_{\text {short }}$ & $10^{-5}$ & $10^{-1}$ & Decay rate of $C_{\text {short }}$ \\
$\beta_{\text {long }}$ & $10^{-10}$ & $10^{-3}$ & Decay rate of $C_{\text {long }}$ \\
$\lambda_{\text {short }}$ & 1 & 6 & Chemoattractant response to $C_{\text {short }}$ \\
$\lambda_{\text {long }}$ & 1 & 6 & Chemoattractant response to $C_{\text {long }}$ \\
$\lambda_{\text {short }}$ & 1 & 6 & Chemoattractant response to $C_{\text {short }}$ \\
$\Phi$ & 0 & 6 & Bonding strength \\
$\Gamma$ & 0 & 6 & Shoving strength \\
$T$ & 0 & 6 & Tight junction to boundary strength \\
\hline
\end{tabular}




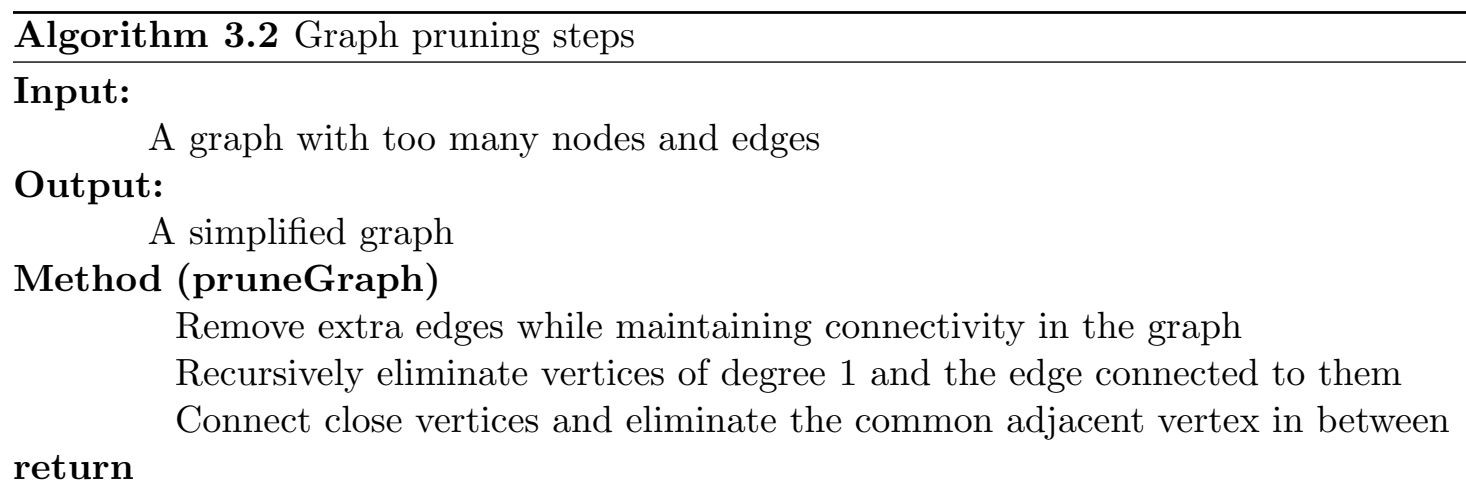

and large for calculations; therefore, it was pruned to better represent the network of cells in the model. To do so, multiple graph manipulation algorithms were used and the pruning was performed in a number of steps for a suitable result. Algorithm 3.2 shows the pruning steps taken to simplify the graph.

Pruning the graph was not possible without visual access to it. However, no suitable graph visualization tool which could satisfy out requirements, especially the maintenance of the node locations were found. Therefore, a program was developed to create the graph in the Virtual Reality realm. Figures 3.1 and 3.2 show illustrations of two sample graphs built and pruned during the simulations.

If the pruned graph lacked a connection from the left to the right pipe cells, no solutes could flow inside the vascular cells; hence, a fitness amount of zero was returned to the GA fitness function. Otherwise, a new simulation in cDynomics started based on the current setting. However, the only difference would be that the the cell factory was set to the active state. This meant that if the base cells were provided with sufficient nutrients, could enter an active producing state and turn green. The duration of this phase was again set in the protocol file. The major output of this phase was the text file containing the amount of metabolic material produced in the culture which was returned to the GA.

Once the GA receives fitness values from all participating members of the population, it applied the previously mentioned genetic operators towards evolution of the population. The instance with a higher product amount, has a higher fitness value and is more likely to 


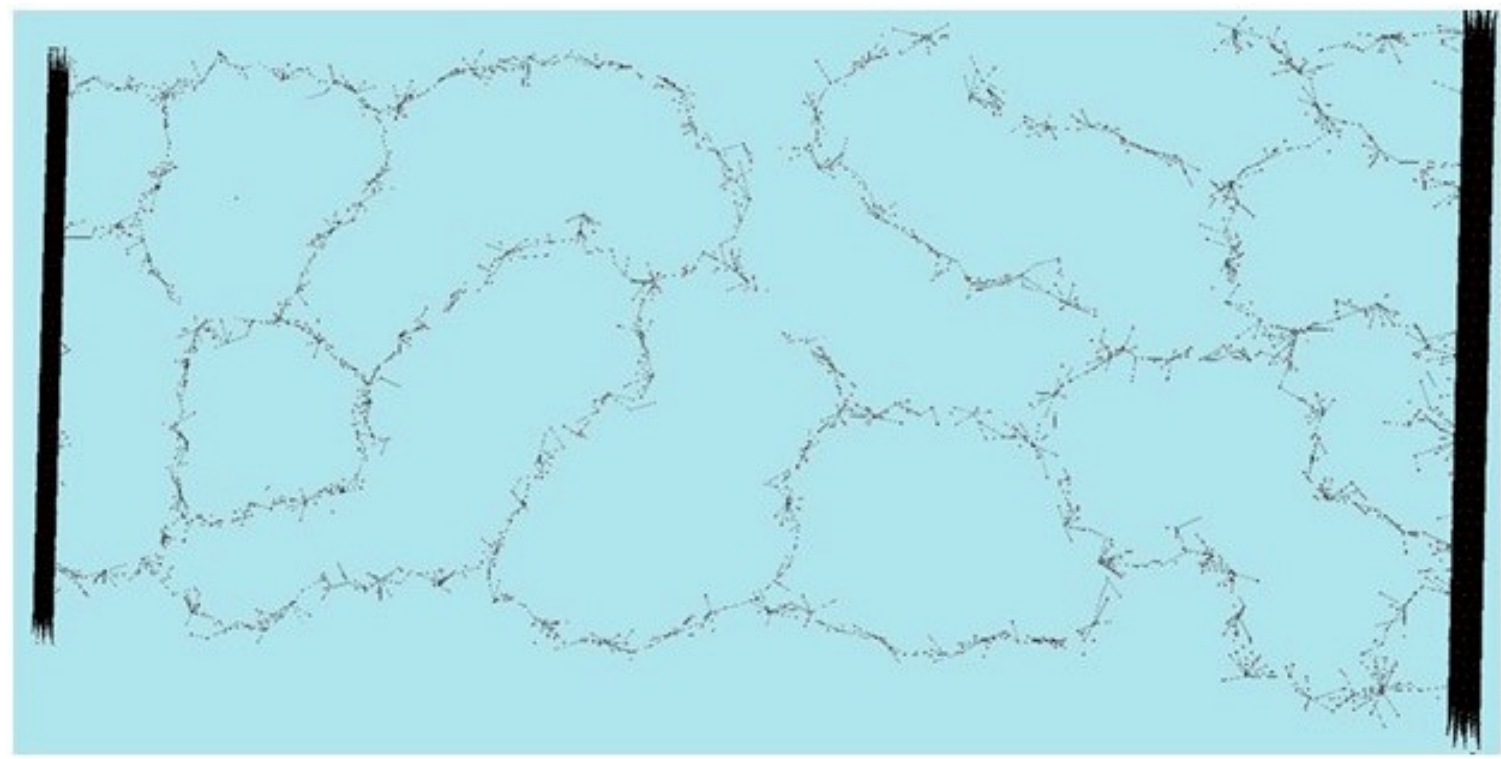

(a)

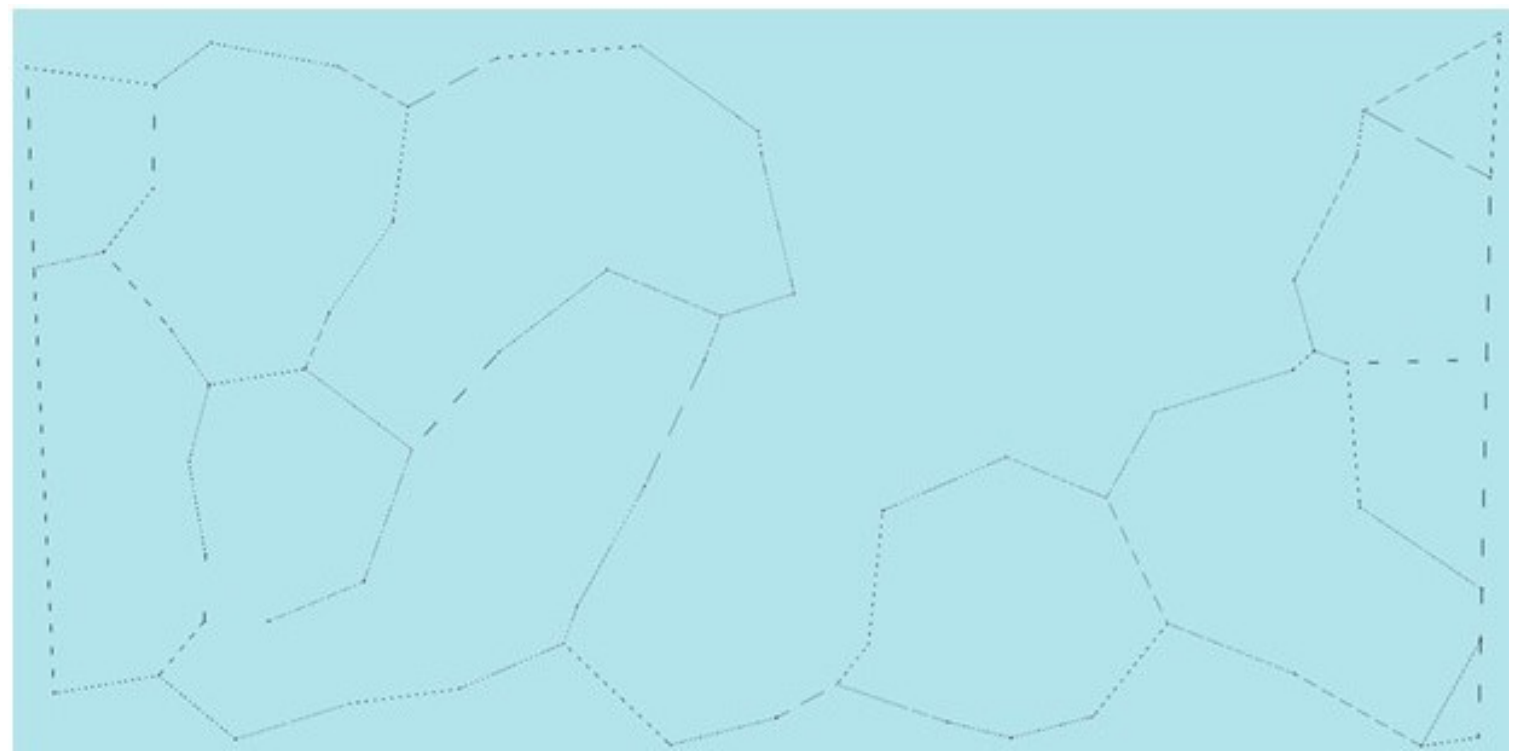

(b)

Fig. 3.1: A sample graph during pruning: Figure 3.1a shows a sample graph built based on the vascular network and experienced one level of simplification. Figure 3.1b shows the same graph after pruning is over and extra nodes and edges have been removed.

be stored for future generations of the GA.

The ending point of a GA can be either reaching a certain fitness value or specific number of iterations. In the case of our experiment, the GA ran for 1000 iteration. 


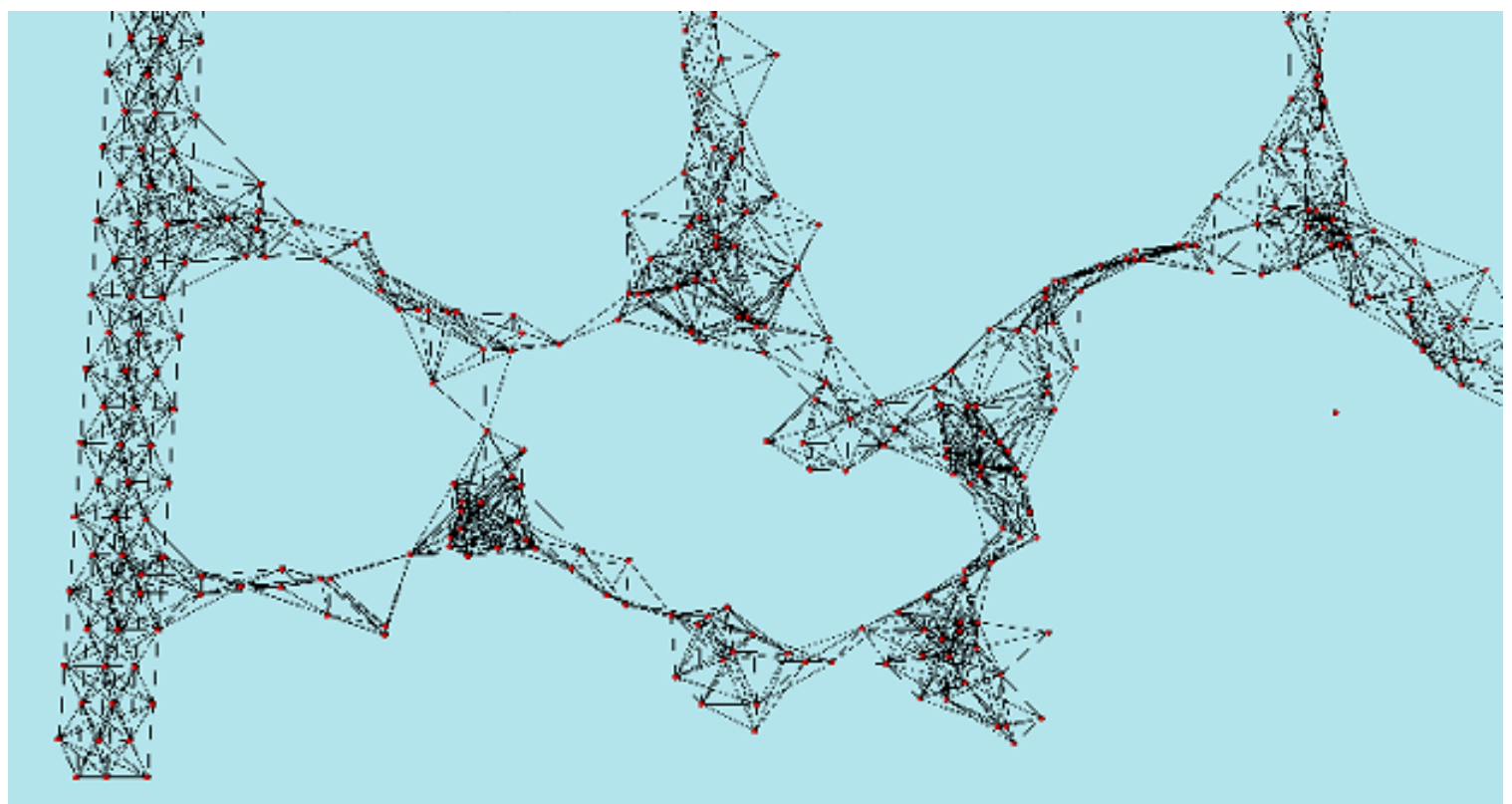

(a)

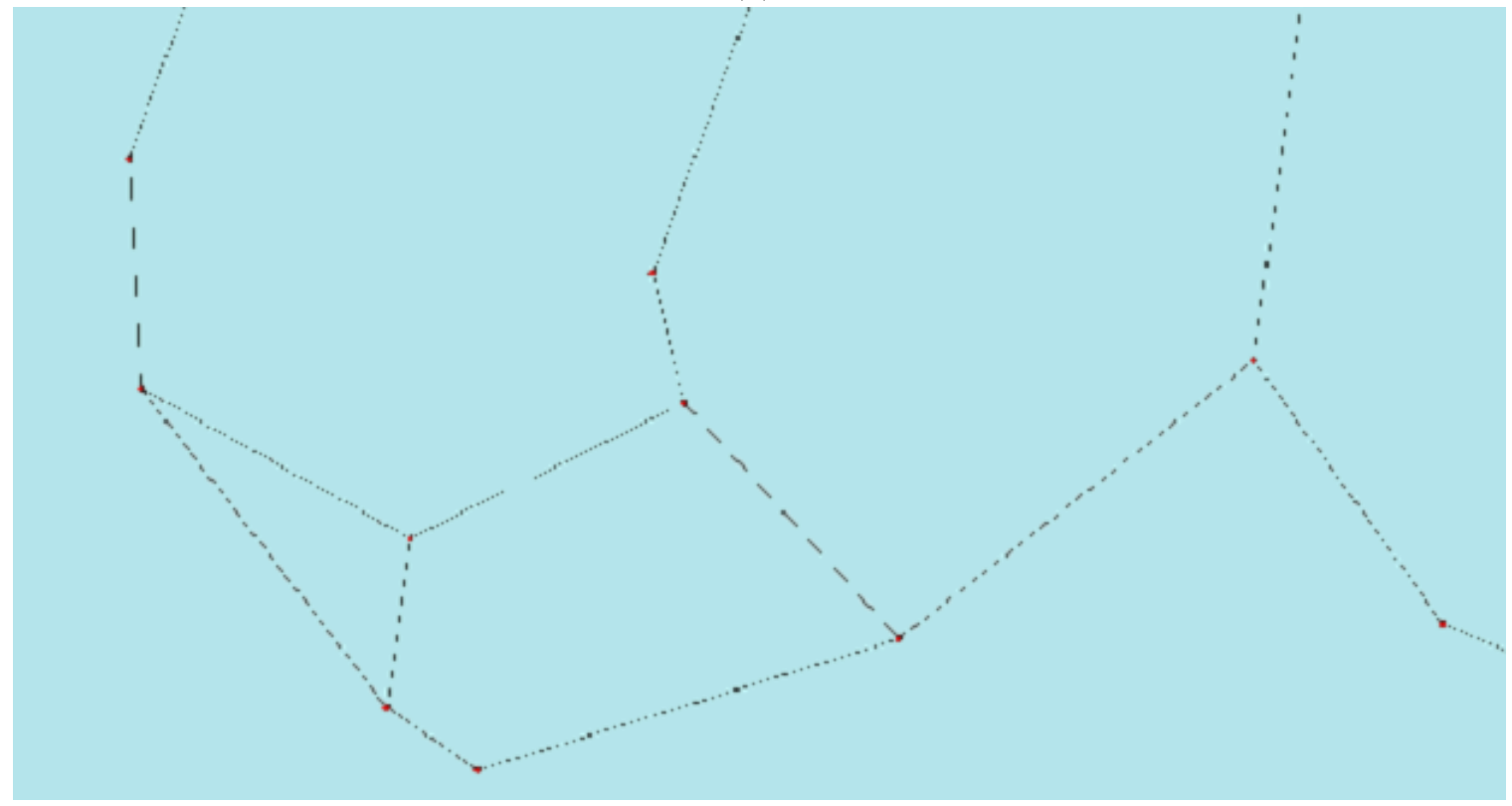

(b)

Fig. 3.2: A closer look at a sample graph during pruning: Figure 3.2a shows a close-up of a section in the graph whereas figure $3.2 \mathrm{~b}$ shows the same corner in the same graph after the pruning is over.

\subsection{Experimental Set-up}

Two sets of experiments were performed which had differences in the model variables and population size. The self organizing vascular models in the experiments had seven and 
ten parameters with unknown values, respectively. The parameters are shown in Tables 3.1 and 3.2. The best fit for these parameters was found using a genetic algorithm from jGAP $[17]$.

The parameters which were selected in this study, were used as genes in individual chromosomes. The initial population had a size of three and five for the primary and expanded experiments, respectively and was kept fixed during the evolution. Although a population consisting of a higher number of members would increase the reliability of the model, limitations around time and resources effected the size decision. However, many GA instances were run to increase reliability; i.e. the program was run independently on a few machines for better comparison of the best output. Totally, the number of instances for each experiment was 20 . The population was constructed by random selection of parameter values from the ranges shown in Tables 3.1 and 3.2 for the two experiments.

As the population evolves during runs of the genetic algorithm, each individual is evaluated using the parameter values encoded in the genes used to run the vascular model (see Section 2.1 for more details). The resulting vascular network is then evaluated as described in Section 2.4, and the fitness is assigned based on the metabolic production. Next, genetic operators were run on the population followed by a re-evaluation of the population that now contained new individuals. Finally, selection was applied to obtain the new population for the next round of evolution. Crossover and mutation operators were included in the genetic algorithm, with the crossover operator applied to $35 \%$ of the population per generation and the mutation operator was applied to each gene with a probability of $1 / 12$. An elitist ranking selector cloned the top $\% 90$ of the population, with the remaining $10 \%$ of the population filled with the same individuals in the order of fitness. The results presented in this paper were obtained using 1000 steps of evolution.

Initial runs of the algorithm were performed on a two dimensional cellular culture. Its height was set to $0.768 \mathrm{~mm}$ while the width was fixed at $1.536 \mathrm{~mm}$. The total number of supported and vascular cells were 4200 and 1800, respectively where vascular cells contributed to 30 per cent of total number of cells. A total of 384 circulatory cells simulating 
the external delivery and metabolic product extraction system is implemented as two identical columns on two sides of the cellular culture. The number of all mentioned cells were kept constant through the experiment. The simulations started with a random distribution of supported and vascular cells inside the culture. Sixteen simulated hours are allocated for network development, after which the networks quality is evaluated. If no path of vessels is found that connects the source to the sink, then the fitness score is set at zero. Otherwise, the fitness score of the network is estimated by measuring the metabolic productivity of the supported cells while simulating the operation of the vascular network for two simulated hours. This process is described in detail in Section 2.4. 


\section{CHAPTER 4}

\section{RESULTS}

\subsection{Results}

\subsubsection{First Experiment}

Figure 4.1 shows one of the GAs in experiment one which led to the best case fitness of the networks produced during the running of the genetic algorithm. The algorithm starts with random values for the chosen parameter within the given ranges. As shown in the graph, the GA is unsuccessful in creating a productive cell culture for the first several evolutions. However, once it finds a parameter set leading to a functioning network, it continually improves the quality of solutions. After 1,000 iterations, the highest fitness level was $537.66 \mu \mathrm{g}$ of metabolic product. The parameter values contributing to the best case are shown in Table 4.1.

Figure 4.2 shows some sample cell cultures produced by the genetic algorithm during its evolution. As it is seen in the figure, some arrangements of the vascular cells do not form a network for the flow of material and thus no production is obtained in the system. Figure 4.2a depicts a cell culture with most of the vascular cells accumulated beside the circulatory cells on the sides and occasional formations in the middle. This can be a result of high chemotactic response to $\mu_{l o n g}$ and $k$.

Table 4.1: The best parameter values found by the GA in initial experiment

\begin{tabular}{lll}
\hline Parameter & Value & Description \\
\hline \hline$k$ & $6.78 \times 10^{-3}$ & Monod-kinetic coefficient \\
$\mu_{\text {short }}$ & 5.12 & Secretion rate of $C_{\text {short }}$ \\
$\mu_{\text {long }}$ & 6.10 & Secretion rate of $C_{\text {long }}$ \\
$\beta_{\text {short }}$ & $67.3 \times 10^{-3}$ & Decay rate of $C_{\text {short }}$ \\
$\beta_{\text {long }}$ & $7.41 \times 10^{-6}$ & Decay rate of $C_{\text {short }}$ \\
$\lambda_{\text {short }}$ & 2.69 & Chemoattractant response to $C_{\text {short }}$ \\
$\lambda_{\text {long }}$ & 1.99 & Chemoattractant response to $C_{\text {long }}$ \\
\hline
\end{tabular}




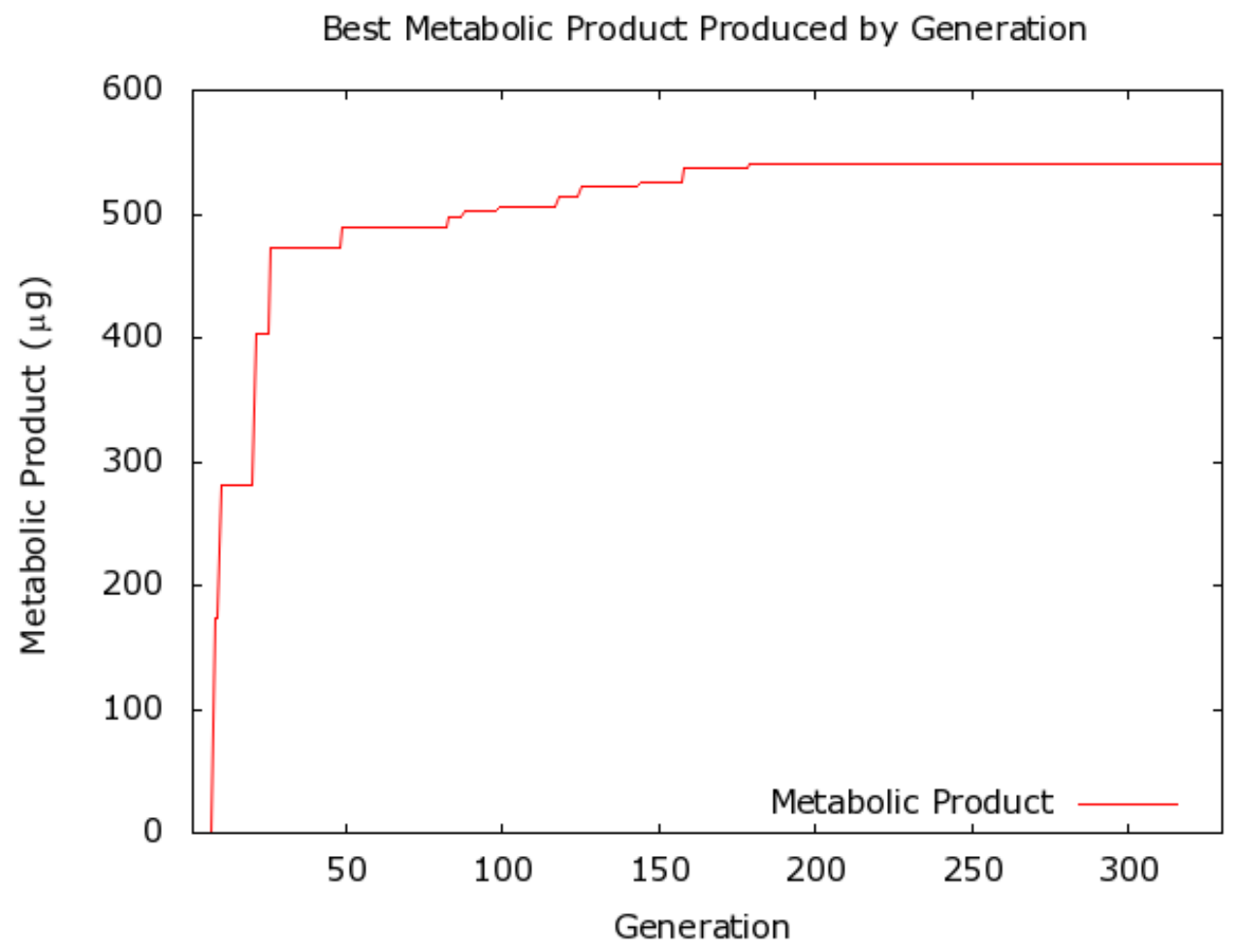

Fig. 4.1: A graph that visualizes how the amount of cell culture production improves over generations of the genetic algorithm in an instance run in the first experiment.

Figures $4.2 \mathrm{~b}$ and $4.2 \mathrm{~d}$ both demonstrate scattered and disconnected lines of vascular cells floating among supported cells. Such arrangements result from a low amount of $\mu_{\text {long }}$ but a high amount of $\beta_{\text {short }}$. It should be mentioned that the cell culture in Figure $4.2 \mathrm{~d}$ has a higher $\beta_{\text {short }}$ than the one in $4.2 \mathrm{~b}$ which accounts for the thicker lines of vascular cells. On the other hand, Figure 4.2c shows another unsupported cell culture with several cycles

Table 4.2: Parameter table corresponding to cell cultures depicted in Figures 4.2 and 4.3

\begin{tabular}{c||c||c||c||c||c||c||c}
\hline Culture & $\lambda_{\text {short }}$ & $\lambda_{\text {long }}$ & $\mu_{\text {short }}$ & $\mu_{\text {long }}$ & $\beta_{\text {short }}$ & $\beta_{\text {long }}$ & $k$ \\
\hline \hline Figure 4.2a & 1.28 & 4.09 & 5.90 & 6.58 & $6.79 \times 10^{-2}$ & $5.96 \times 10^{-4}$ & $2.64 \times 10^{-3}$ \\
Figure 4.2b & 1.28 & 5.92 & 5.90 & 5.21 & $6.79 \times 10^{-2}$ & $8.54 \times 10^{-4}$ & $8.96 \times 10^{-3}$ \\
Figure 4.2c & 1.11 & 1.22 & 6.69 & 4.50 & $9.90 \times 10^{-2}$ & $2.06 \times 10^{-4}$ & $1.49 \times 10^{-3}$ \\
Figure 4.2d & 5.73 & 1.22 & 6.69 & 4.50 & $8.14 \times 10^{-2}$ & $5.70 \times 10^{-4}$ & $2.17 \times 10^{-3}$ \\
Figure 4.3 & 2.69 & 1.34 & 3.85 & 6.10 & $3.23 \times 10^{-2}$ & $7.68 \times 10^{-5}$ & $2.64 \times 10^{-4}$ \\
\hline
\end{tabular}




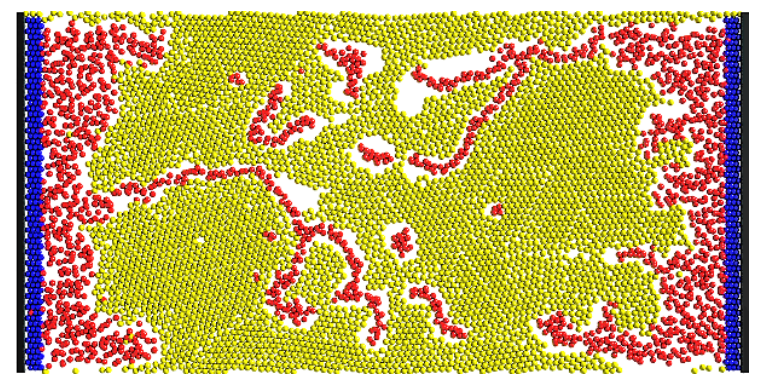

(a)

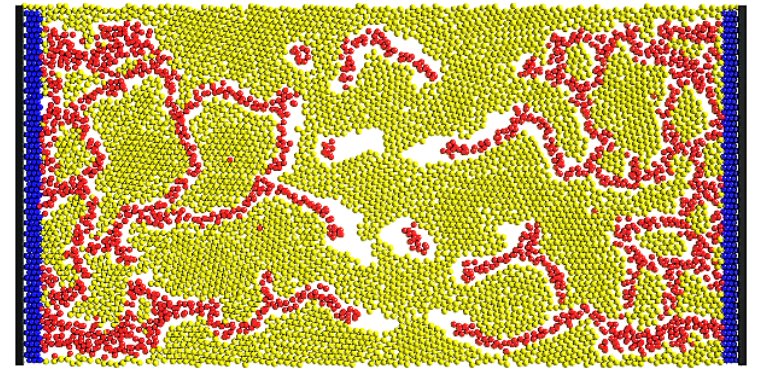

(c)

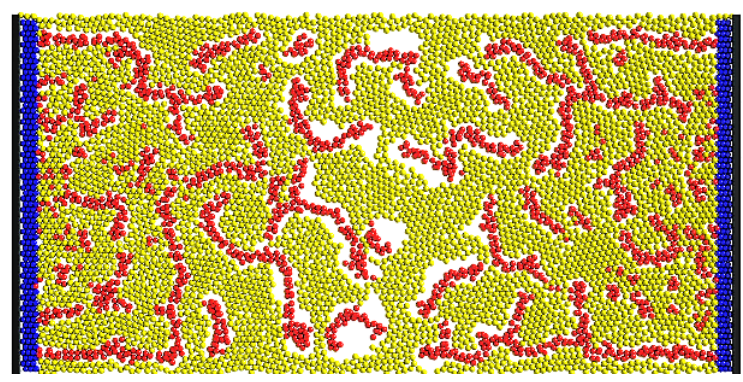

(b)

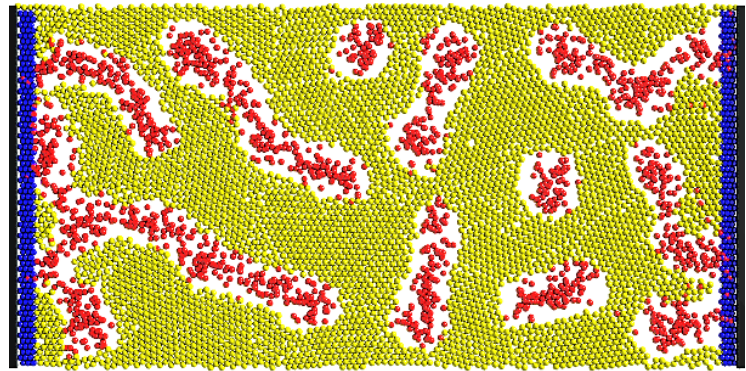

(d)

Fig. 4.2: Sample results of the GA during its evolution. These depict unsuccessful cell cultures at creating a consistent vascular network for the flow of nutrient and product

of vascular cells but no path connecting the source to the sink. This result follows from low $\lambda_{\text {short }}$ and high $\lambda_{\text {long }}$ and $\mu_{\text {long }}$.

Figure 4.3 depicts cellular cultures found by the GA that have successfully formed networks of vascular cells and reached the highest amount of production. No patches of yellow non-producing cells are present in these cell cultures. As depicted, vascular cells have successfully formed a number of lacunae that feed all supported cells available in the culture and move waste and products away. More lacunae of vascular cells means availability of nutrients to more supported cells and therefore, higher production. Investigating the parameters leading to these solutions reveal an average amount of $\lambda_{\text {short }}$ and $\lambda_{\text {long }}$ and low amounts of $\mu_{\text {short }}, \mu_{\text {long }}, \beta_{\text {short }}, \beta_{\text {long }}$ and $k$. There are few unconnected branches stretching from a few points which given a larger time frame could lead to more lacunae and even a higher production. The exact amount of each parameter for cultures depicted in Figures 4.2 is given in Table 4.2. For comparison purposes, the parameter values for Figure 4.3(a) is also given in the same table. 


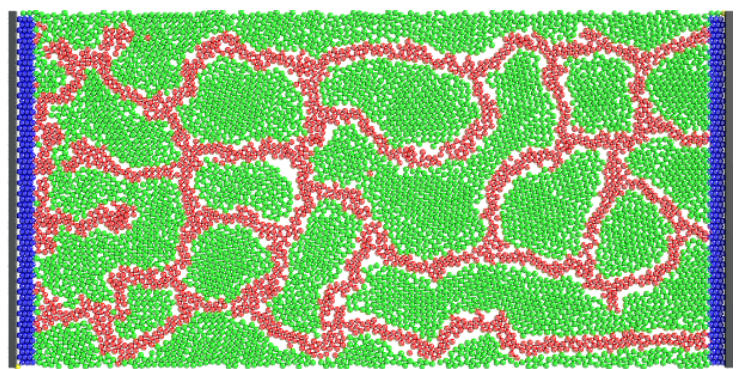

(a)

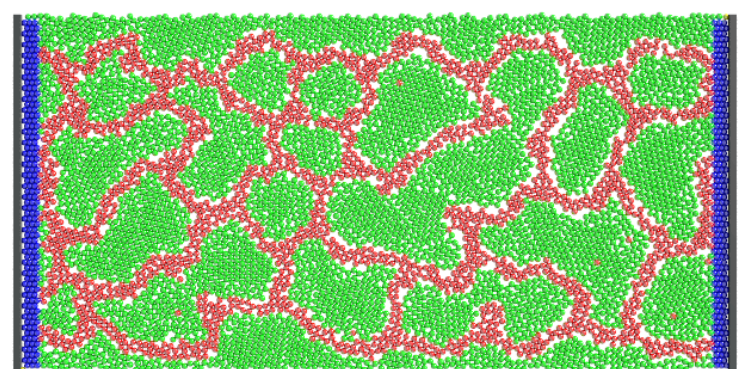

(b)

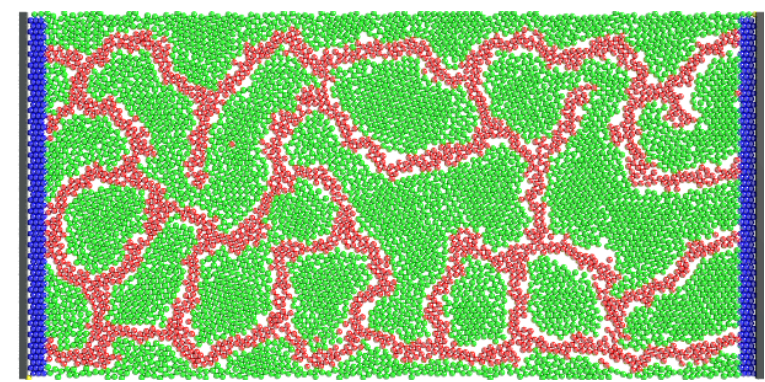

(c)

Fig. 4.3: A sample of best solutions identified by the genetic algorithm during evolution. These results depict vascular networks that have successfully nourished the supported cells along with removing their product and waste

\subsubsection{Expanded Experiment}

Evolution of the GA instance leading to the highest cellular productivity during the experiment with expanded configuration is depicted in Figure 4.4. The maximum amount of production it reached was $828.45 \mu \mathrm{g}$ which is noticeably higher than the result in the initial experiment. In addition, it ran for about a hundred fewer evolution steps due to time limitations; thus, reaching a high result in this stage looks promising. Similar to the previous GA instance, this one starts by zero product amount for the first 40 evolutions until discovering a higher than zero productivity.

Values discovered for the highest productivity are presented in Table 4.3. Investigating parameter values during the evolution of the GA reveal that no specific relationship between values can be identified at this stage. Although at some GA solutions pertaining to high productivity noticeably higher values of $\mu_{\text {short }}$ and $\lambda_{\text {short }}$ relative to $\mu_{\text {long }}$ and $\lambda_{\text {long }}$ are observed, other high productivity solutions such as the one presented in table 4.3 do not 


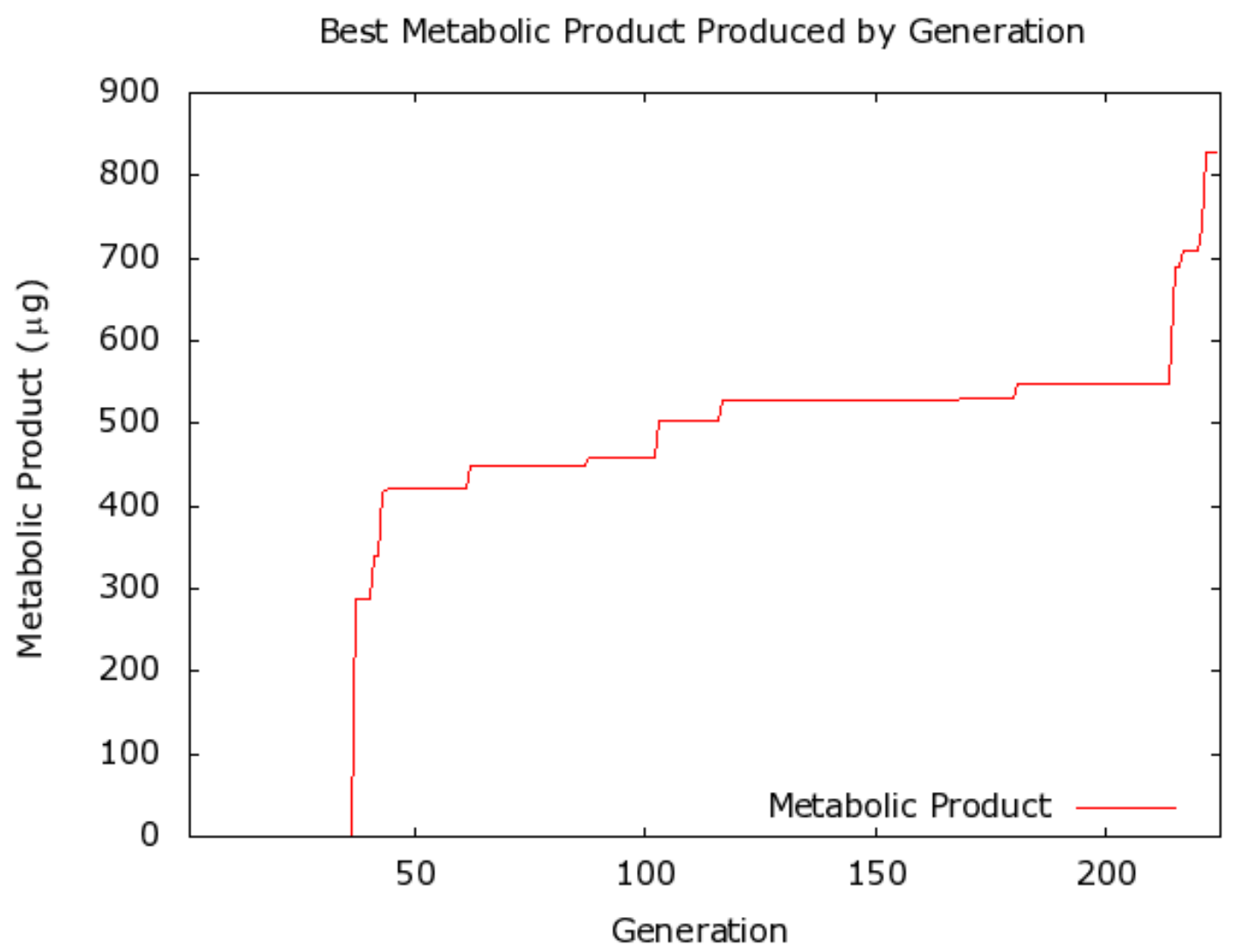

Fig. 4.4: A visualization of cell culture productivity improvement over generations of the genetic algorithm in the expanded experiment

Table 4.3: The best parameter values found by the GA

\begin{tabular}{lll}
\hline Parameter & Value & Description \\
\hline \hline$k$ & $9.99 \times 10^{-3}$ & Monod-kinetic coefficient \\
$\mu_{\text {short }}$ & 6.84 & Secretion rate of $C_{\text {short }}$ \\
$\mu_{\text {long }}$ & 6.10 & Secretion rate of $C_{\text {long }}$ \\
$\beta_{\text {short }}$ & $6.53 \times 10^{-2}$ & Decay rate of $C_{\text {short }}$ \\
$\beta_{\text {long }}$ & $2.32 \times 10^{-5}$ & Decay rate of $C_{\text {short }}$ \\
$\lambda_{\text {short }}$ & 1.42 & Chemoattractant response to $C_{\text {short }}$ \\
$\lambda_{\text {long }}$ & 5.96 & Chemoattractant response to $C_{\text {long }}$ \\
$\Phi$ & 0.94 & Tight junction creation factor \\
$\Gamma$ & 5.35 & Tight junction breakage factor \\
$T$ & 2.48 & Tight junction to boundary strength \\
\hline
\end{tabular}

show such a relation. In addition, high amounts of $\Phi$ and $T$ and a low amount of $\Gamma$ seem necessary for avoidance of discreet clots of vascular cells; however, some successful solutions of the GA do not comply with this hypothesis.

Two of the simulations with the highest simulated culture productivity are shown in 
Figure 4.5. These images show robust networks of vascular cells which have improved the factory efficiency enabling high amounts of production. They are quite similar to depictions of the most successful cell factories in Figure 4.3 whereas the number of minimal cycles of cells and branches have increase. We believe more branching and a higher number of lacunae provides access to a broader range of cells due to distances among vascular and support cells. Therefore, more and thinner branches are favorable to a lower number of thick branches in the simulated culture.

Four sample images during the evolution path of the GAs have been provided in Figure 4.6. Parameter values pertaining to these illustrations are also presented in Table 4.4.

The discreet lumps in Figure 4.6a have formed due to high amounts of $\lambda_{\text {short }}, \mu_{\text {short }}$, $\Gamma$ and $T$ but a low amount of $\beta_{\text {short }}$. This causes the vascular cells to connect to each other in small gatherings and not move away. Higher amounts of $\lambda_{\text {long }}$ and $\mu_{\text {long }}$ than $\lambda_{\text {short }}$ and $\mu_{\text {short }}$, respectively and also a very high $\beta_{\text {short }}$ in the factory depicted in figure $4.6 \mathrm{~d}$, is the reason behind vascular cells accumulating beside circulatory cells on the sides. Moreover, substantially higher tight junction creation factors than tight junction destroy factor has led to vascular cells' large connectivity areas. Dispersed cells drawn towards the middle of the world in Figure 4.6c are mainly resulted from low levels of $\mu_{\text {short }}, \Phi$ and $T$ but a higher level of $\Gamma$. Among these illustrations, Figure 4.6b shows the potential to create a vascular network given a longer time span. This can be explained by the very high amounts of $\mu_{\text {long }}$

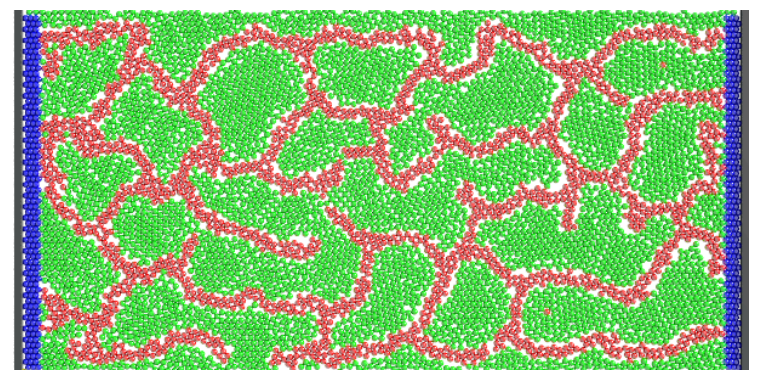

(a)

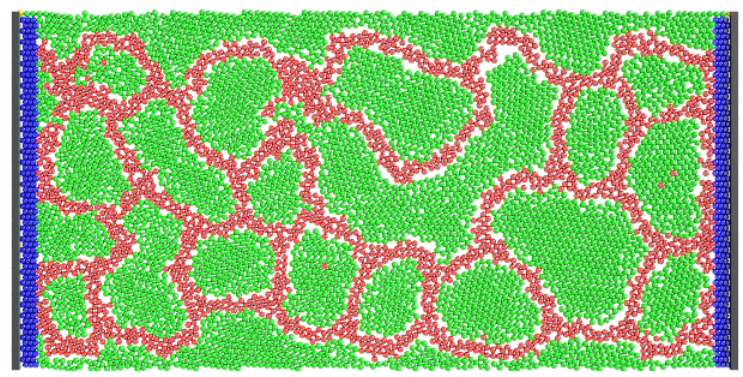

(b)

Fig. 4.5: Two of the best solutions identified by the genetic algorithm during evolution. The vascular networks are well distributed among supported cells, successfully nourishing them. 


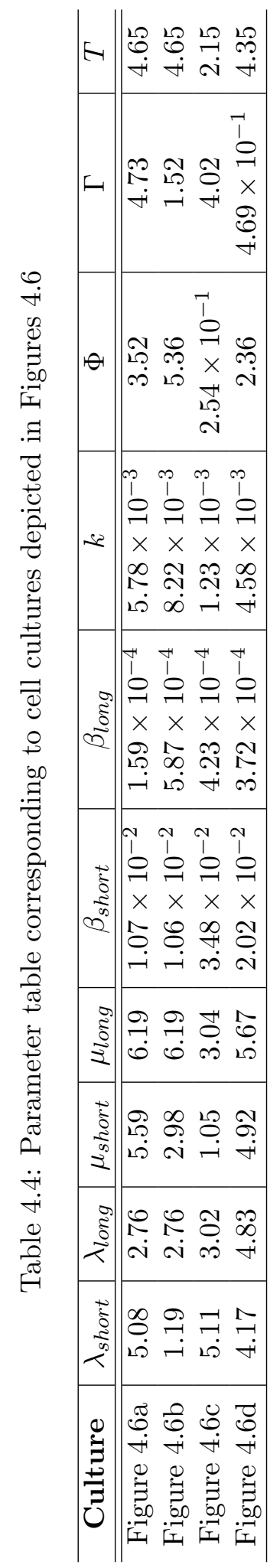




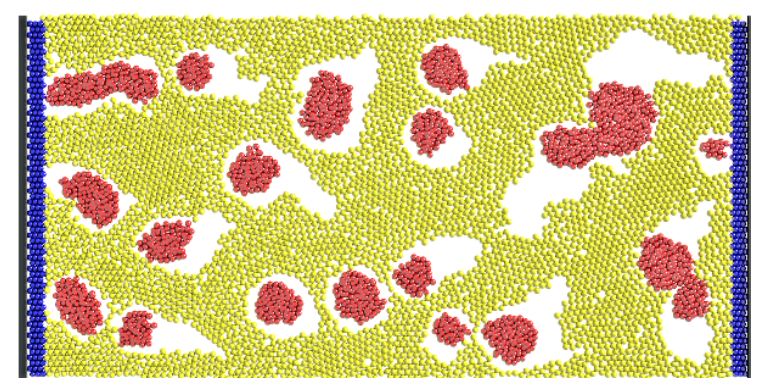

(a)

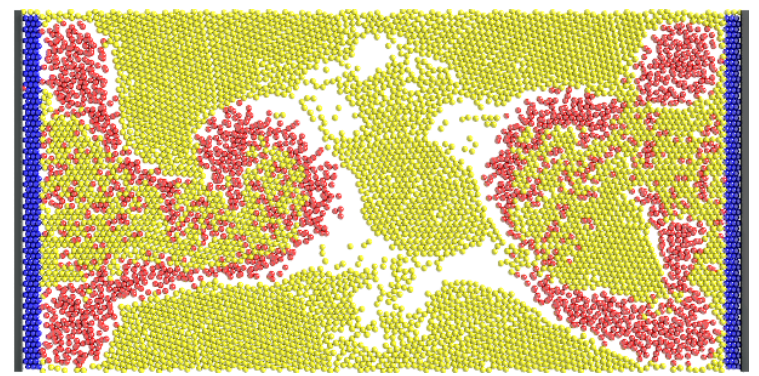

(c)

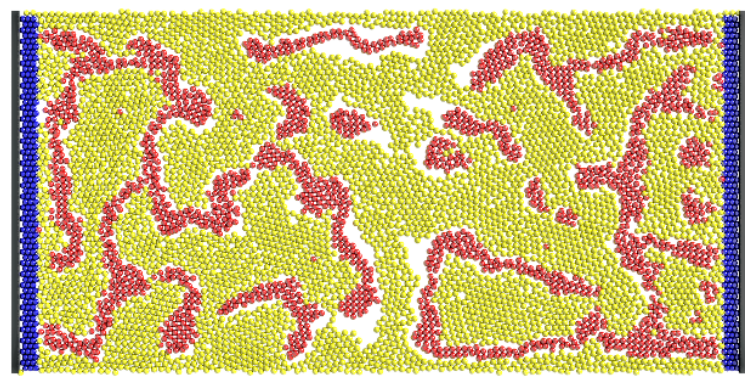

(b)

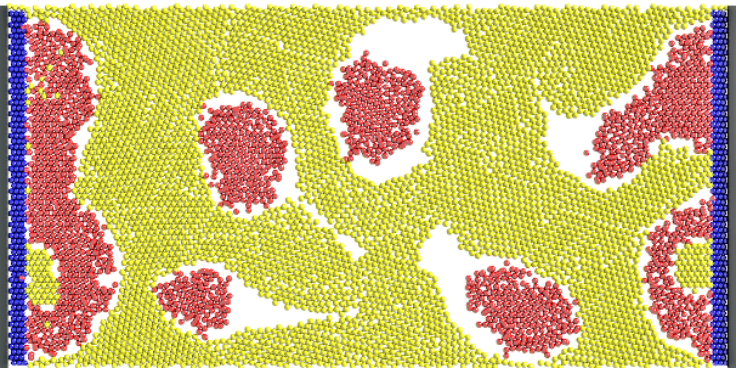

(d)

Fig. 4.6: Illustration of some solutions which have not been successful in creating a reliable network of cells for nourishing the support cells and removing their products

and $k$ and low amounts of $\mu_{\text {short }}$ and $\lambda_{\text {short }}$. 


\section{CHAPTER 5}

\section{DISCUSSION AND CONCLUSION}

This work explored a solution to the problem faced by bioengineers to determine those cellular mechanisms to target for modification when improving vascularization for synthetic tissue engineering. The method integrated a hybrid particle-based model of vascularization as a fitness function with a genetic algorithm applied to optimize the search space of model parameter values. The method is generic in that models of different biological systems could be substituted for vascularization and the fitness function likewise changed to reflect alternative objectives, such as minimizing vascular function to combat tumor growth in cancer $[18]$.

In this study, the search space was initially reduced to only consider parameters that influence the chemotactic mechanism of the vascular cells since we believed that they would have the most tangible effect on vascularization. On the other hand, previous work with the model had identified the importance of adhesion and tight junction formation in robust network formation. As a result, the experiment was repeated with addition of three more parameters targeting tight junction and adhesive quality of the cells.

The model presented for bio-reactor simulation involved cellular characteristics such as biomass, division, death, mutation, etc. The cells interact via physical forces of bonding and shoving. They also get involved in different reactions, produce extracellular polymeric substances (EPS) to hold them together and are influenced by chemotactic secretions, the primary reason behind formation of vessels. The model is capable of illustrating suitable productivity if a robust network of cells supports the primary cells in the simulation culture.

Genetic algorithms have proven to be strong optimization tools in case of complex problems such as non-convex, stochastic cases. They are developed by inspiring from natural genetic mechanisms replicating some functionality like natural selection, mutation and crossover. Thus, we chose to approach our optimization problem with a genetic algorithm which 
was provided as a software package bundle. The two sets of seven and ten parameters each were selected as the algorithm variables and the objective function was the productivity of the microbial factory.

Two experiments were performed to discover optimal parameter values in the model. Both experiments were successful in identifying values required for robust vascular network structures. However, the second experiment, which was an expansion to the initial showed a significantly better result compared to it. In the meanwhile, it should be considered that with a possibly broader time period, it may display even better results.

The main issue regarding solutions which adopt genetic and evolutionary algorithms for optimization is the uncertainty in finding the optimal values due to the algorithm's blindness to values it has not tried. Therefore, to insure results' correctness, it is required to rum the model for many times. Given the modeling framework employed here [9], each fitness evaluation takes at least four hours of CPU time running on a $3.6 \mathrm{GHz}$ machine which makes it currently impractical to run too many iterations of the genetic algorithm. Recently two fast large-scale simulation systems have been developed by Ghaffarizadeh et al. [19] and Biocellion [20]. Both these systems implement an individual-based approach similar to $c$ DynoMiCs employed here. Biocellion is implemented as a distributed architecture executable on the Cloud [21] and is capable of simulating complex 3D models of billions of cells in a matter of a few hours. Biocellion has the potential to simulate vascularization quickly enabling the optimization of more complex bioengineered multicellular systems.

The principle challenge in bringing this work to practicality is to operationalize the link between model parameters and actual genetic sequences. If this can be achieved, then the output of the optimization process could be directly mapped to engineering targets in the genome. In traditional bioengineering where products are produced in biofactories [22], [23], targets are identified through analysis of detailed metabolic models of microbial cells, such as [24]. The functioning of mammalian cells is less understood, particularly mechanisms such as chemotaxis that are unrelated to metabolic processes, which can be modeled using flux balance analysis. 
The key to operationalizing bioengineering of multicellular systems is to utilize multiscale models of the biological system under study. Multiscale models link the mechanisms acting at different spatial and temporal scales together into an integrated system where changes in expression and regulation of genes are manifested in large-scale multicellular outcomes [25], [26] [27]. If such detailed models were employed, the high-level abstract parameters of this work would be replaced by specific genetic mechanisms resulting in solutions being mapped directly to genetic targets.

This work has shown the genetic algorithms are powerful enough to optimize complex biological systems where there is a non-linear relationship between the parameters being searched and the fitness of the solutions. The method can be applied to other models of other biological systems with alternative objectives.

Many challenges exist before this optimization approach can be utilized to support the process of bioengineering tissues. Two principle challenges are (a) the speed of simulations needs to be increased many orders of magnitude to expand the parameter space and fidelity of the models, (b) a means of mapping the parameter space searched by the GA to operational genome modifications needs to be realized. 


\section{REFERENCES}

[1] R. Monahan-Earley, A. M. Dvorak, and W. C. Aird, "Evolutionary origins of the blood vascular system and endothelium." Journal of thrombosis and haemostasis : JTH, vol. 11 Suppl 1, pp. 46-66, Jun. 2013. [Online]. Available: http://view.ncbi.nlm.nih.gov/pubmed/23809110

[2] R. M. H. Merks, E. D. Perryn, A. Shirinifard, and J. A. Glazier, "ContactInhibited Chemotaxis in De Novo and Sprouting Blood-Vessel Growth," PLoS Comput Biol, vol. 4, no. 9, pp. e1000163+, Sep. 2008. [Online]. Available: http://dx.doi.org/10.1371/journal.pcbi.1000163

[3] T. Kaully, K. Kaufman-Francis, A. Lesman, and S. Levenberg, "VascularizationThe Conduit to Viable Engineered Tissues," Tissue Engineering Part B: Reviews, vol. 15, no. 2, pp. 159-169, Mar. 2009. [Online]. Available: http://dx.doi.org/10.1089/ten.teb. 2008.0193

[4] M. Lovett, K. Lee, A. Edwards, and D. L. Kaplan, "Vascularization Strategies for Tissue Engineering," Tissue Engineering Part B: Reviews, vol. 15, no. 3, pp. 353-370, Jun. 2009. [Online]. Available: http://dx.doi.org/10.1089/ten.teb.2009.0085

[5] T. Takebe, N. Koike, K. Sekine, R. Fujiwara, T. Amiya, Y.-W. W. Zheng, and H. Taniguchi, "Engineering of human hepatic tissue with functional vascular networks." Organogenesis, vol. 10, no. 2, pp. 260-267, 2014. [Online]. Available: http://view.ncbi.nlm.nih.gov/pubmed/24451152

[6] J. M. Melero-Martin, M. E. De Obaldia, S.-Y. Kang, Z. A. Khan, L. Yuan, P. Oettgen, and J. Bischoff, "Engineering Robust and Functional Vascular Networks In Vivo With Human Adult and Cord BloodDerived Progenitor Cells," Circulation Research, vol. 103, no. 2, pp. 194-202, Jul. 2008. [Online]. Available: http://dx.doi.org/10.1161/circresaha.108.178590

[7] H. Z. Miguel Anjos, "Optimization and engineering applications," 2016.

[8] C. L. Reiber and I. J. McGaw, "A Review of the Open and Closed Circulatory Systems: New Terminology for Complex Invertebrate Circulatory Systems in Light of Current Findings," International Journal of Zoology, vol. 2009, pp. 1-8, 2009. [Online]. Available: http://dx.doi.org/10.1155/2009/301284

[9] L. A. Lardon, B. V. Merkey, S. Martins, A. Dötsch, C. Picioreanu, J.-U. Kreft, and B. F. Smets, "iDynoMiCS: next-generation individual-based modelling of biofilms," Environmental Microbiology, vol. 13, no. 9, pp. 2416-2434, Sep. 2011. [Online]. Available: http://dx.doi.org/10.1111/j.1462-2920.2011.02414.x

[10] J. Adler, "Chemotaxis in Escherichia coli," Cold Spring Harbor Symposia on Quantitative Biology, vol. 30, pp. 289-292, Jan. 1965. [Online]. Available: http://dx.doi.org/10.1101/sqb.1965.030.01.030 
[11] S. P. Sutera and R. Skalak, "The History of Poiseuille's Law," Annual Review of Fluid Mechanics, vol. 25, no. 1, pp. 1-20, 1993. [Online]. Available: http://dx.doi.org/10.1146/annurev.fl.25.010193.000245

[12] D. Davis, A. Doloman, G. J. Podgorski, E. Vargis, and N. S. Flann, "Exploiting Self-organization in Bioengineered Systems: A Computational Approach," Frontiers in Bioengineering and Biotechnology, vol. 5, Apr. 2017. [Online]. Available: http://dx.doi.org/10.3389/fbioe.2017.00027

[13] O. Bernard, G. Bastin, C. Stentelaire, L. Lesage-Meessen, and M. Asther, "Mass balance modeling of vanillin production from vanillic acid by cultures of the fungus Pycnoporus cinnabarinus in bioreactors," Biotechnol. Bioeng., vol. 65, no. 5, pp. 558-571, Dec. 1999. [Online]. Available: http://dx.doi.org/10.1002/(sici) 1097-0290(19991205)65:5\%3C558::aid-bit9\%3E3.0.co;2-a

[14] K. Han and O. Levenspiel, "Extended monod kinetics for substrate, product, and cell inhibition," Biotechnol. Bioeng., vol. 32, no. 4, pp. 430-447, Aug. 1988. [Online]. Available: http://dx.doi.org/10.1002/bit.260320404

[15] O. Levenspiel, "The monod equation: A revisit and a generalization to product inhibition situations," Biotechnol. Bioeng., vol. 22, no. 8, pp. 1671-1687, Aug. 1980. [Online]. Available: http://dx.doi.org/10.1002/bit.260220810

[16] S. Aiba, M. Shoda, and M. Nagatani, "Kinetics of product inhibition in alcohol fermentation," Biotechnol. Bioeng., vol. 10, no. 6, pp. 845-864, Nov. 1968. [Online]. Available: http://dx.doi.org/10.1002/bit.260100610

[17] K. Meffert. (2011) Java genetic algorithms package. [Online]. Available: http: //www.jgap.sourceforge.net

[18] A. W. Mahoney, G. J. Podgorski, and N. S. Flann, "A Multi-Objective Optimization Based-Approach for Discovering Novel Cancer Therapies," IEEE/ACM Transactions on Computational Biology and Bioinformatics, vol. Preprint, Apr. 2010.

[19] A. Ghaffarizadeh, S. H. Friedman, and P. Macklin, "Agent-based simulation of large tumors in 3-D microenvironments," bioRxiv, p. 035733, 2015.

[20] S. Kang, S. Kahan, J. McDermott, N. Flann, and I. Shmulevich, "Biocellion: accelerating computer simulation of multicellular biological system models." Bioinformatics (Oxford, England), vol. 30, no. 21, pp. 3101-3108, Nov. 2014. [Online]. Available: http://dx.doi.org/10.1093/bioinformatics/btu498

[21] I. A. Hashem, I. Yaqoob, N. B. Anuar, S. Mokhtar, A. Gani, and S. Ullah Khan, "The rise of big data on cloud computing: Review and open research issues," Information Systems, vol. 47, pp. 98-115, Jan. 2015. [Online]. Available: http://dx.doi.org/10.1016/j.is.2014.07.006

[22] R. Sharma, Y. Chisti, and U. C. Banerjee, "Production, purification, characterization, and applications of lipases." Biotechnology advances, vol. 19, no. 8, pp. 627-662, Dec. 2001. [Online]. Available: http://view.ncbi.nlm.nih.gov/pubmed/14550014 
[23] J. M. M. van Dijl and M. Hecker, "Bacillus subtilis: from soil bacterium to super-secreting cell factory." Microbial cell factories, vol. 12, 2013. [Online]. Available: http://view.ncbi.nlm.nih.gov/pubmed/23311580

[24] P. D. Karp, M. Latendresse, S. M. Paley, M. K. Q. Ong, R. Billington, A. Kothari, D. Weaver, T. Lee, P. Subhraveti, A. Spaulding, C. Fulcher, I. M. Keseler, and R. Caspi, "Pathway Tools version 19.0: Integrated Software for Pathway/Genome Informatics and Systems Biology," Oct. 2015. [Online]. Available: http://arxiv.org/abs/1510.03964

[25] M. L. Martins, S. C. Ferreira, and M. J. Vilela, "Multiscale models for biological systems," Current Opinion in Colloid \& Interface Science, vol. 15, no. 1-2, pp. 18-23, Apr. 2010. [Online]. Available: http://dx.doi.org/10.1016/j.cocis.2009.04.004

[26] J. A. Joseph Walpole, "Multiscale Computational Models of Complex Biological Systems - Annual Review of Biomedical Engineering," Annual Review of Biomedical Engineering, vol. q5, pp. 137-154, 2013. [Online]. Available: http: //annualreviews.org/doi/abs/10.1146/annurev-bioeng-071811-150104

[27] J. S. Yu and N. Bagheri, "Multi-class and multi-scale models of complex biological phenomena," Current Opinion in Biotechnology, vol. 39, pp. 167-173, Jun. 2016. [Online]. Available: http://dx.doi.org/10.1016/j.copbio.2016.04.002 